\title{
Disjunction and Existence Properties in Inquisitive First-Order Logic
}

\begin{abstract}
Classical first-order logic FO is commonly used to study logical connections between statements, that is sentences that in every context have an associated truthvalue. Inquisitive first-order logic InqBQ is a conservative extension of FO which captures not only connections between statements, but also between questions. In this paper we prove the disjunction and existence properties for InqBQ relative to inquisitive disjunction $\mathbb{V}$ and inquisitive existential quantifier $\bar{\exists}$. Moreover we extend these results to several families of theories, among which the one in the language of FO. To this end, we initiate a model-theoretic approach to the study of InqBQ. In particular, we develop a toolkit of basic constructions in order to transform and combine models of InqBQ.
\end{abstract}

Keywords: Inquisitive logic, Disjunction property, Existence property, Team semantics, Operations on information models, Permutation-based model.

\section{Introduction}

In this paper we prove the disjunction and existence properties for the logic InqBQ, solving a conjecture stated in [3] $\S 4$. To this end, we develop several model-theoretic constructions to study InqBQ and its entailment relation.

\subsection{Motivations}

Classical first order logic (FO) has proven to be a suitable framework to interpret a certain class of sentences, namely statements. If we consider the statements

(a) "The element $c$ has property $P$ ",

(b) "There exists an element with property $P$ ",

clearly (a) implies (b), as witnessed by the entailment $P(c) \vDash^{\text {FO }} \exists x . P(x)$.

Presented by Heinrich Wansing; Received November 29, 2017 
It is interesting to notice that logical relations between questions, as well as relations between questions and statements, also arise naturally. If we consider the questions

(c) "What is an element with property P?",

(d) "What is an element with properties $P$ and $Q$ ?",

we can see for example that (d) determines (c) (given an element with properties $P$ and $Q$, we also have an element with property $P$ ); (c) presupposes (b) (question (c) only has a true answer provided that some element with property $\mathrm{P}$ exists); (a) resolves (c) (knowing that $c$ has property $P$ resolves the issue raised by the question).

As FO is not concerned with questions, these intuitive relations cannot be directly studied by it. The aim of inquisitive first order logic InqBQ ([3]; see also $[5,10]$ ) is to generalize FO by representing questions as formulas and studying the relations between them through the entailment relation. In particular, the relations of determinacy, presupposition and resolution presented above are formalized naturally as a facet of the entailment relation of InqBQ.

In order to represent logical relations between questions as well as statements, InqBQ introduces an information-based generalization of the semantics of FO, the so-called support semantics. The approach adopted is the one of Team Semantics $[1,7,9,11]$ : an information state consists of a collection of models, namely those compatible with a certain piece of information. Intuitively, an information state supports a statement if the statement is implied by the piece of information. And an information state supports a question if the issue raised by the question is resolved by the piece of information. This intuition is formalized by introducing the class of information models, the semantic objects studied by InqBQ, and by defining the support relation as the satisfiability relation for this class.

The approach of representing statements and questions in a uniform way allows to encompass complex sentences, such as conditional questions ("If there exists an element with property $P$, what is an example?"). This broadens significantly the expressive power of FO. The entailment relation of InqBQ then becomes a fundamental tool to represent formally the logical relations introduced above and studying this entailment brings to the fore the logical connections and rules that govern such relations. 


\subsection{Inquisitive Disjunction and Inquisitive Existential Quantifier}

In order to represent questions as formulas, two question-forming operators are added to the syntax of $\mathrm{FO}$, inquisitive disjunction $\mathbb{V}$ and inquisitive existential quantifier $\bar{\exists}$. The role of inquisitive disjunction is to represent alternative questions such as "Does $c$ have property $P$ or $Q$ ?" $(P(c) \Downarrow Q(c))$, while inquisitive existential quantifier introduces existential questions such as "What is an element with property $P ? "(\bar{\exists} x . P(x))$. The semantic account for these two operators is defined in accordance with this interpretation. An information state $s$ supports an alternative question $\varphi \mathbb{V} \psi$ (in symbols

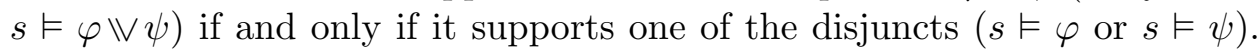
And $s$ supports an existential question $\bar{\exists} x \cdot \varphi(x)$ if and only if it supports an instantiation of the formula $\varphi(x)(s \vDash \varphi(a)$ for some element $a)$.

The intuitive interpretation and the semantics both point to the intuitionistic character of the two operators, which can be thought of as constructive versions of their classical counterparts $\vee$ and $\exists$. Indeed, there is a tight relation between InqBQ and intuitionistic first-order logic, the best known and most studied example of constructive logic (see [2] for detailed discussions).

Exploiting the constructive character of these operators and the connections with intuitionistic logic is of fundamental importance for recognizing the properties of InqBQ entailment relation. In particular, the two properties that are considered the hallmark of constructivism and are well known to hold for intuitionistic logic are the disjunction and existence properties. ${ }^{1}$ Do these properties hold for InqBQ? The positive answer to this question was already conjectured in [3]. Moreover, this result is also suggested by the following intuitive reading of the two properties. If the issue raised by an alternative question is purely logically resolved, then one of the alternatives should be valid (disjunction property). And if the issue raised by an existential question is purely logically resolved, then it is possible to find a term witnessing this resolution (existence property).

\subsection{Results}

In this article we give a positive answer to this conjecture and show that a more general version of the disjunction and existence properties hold:

\footnotetext{
${ }^{1}$ Recall that these two properties do not hold for classical logic. Indeed, the formula $P(c) \vee \neg P(c)$ is an instance of the principle of excluded middle, and so is classically valid; but neither $P(c)$ nor $\neg P(c)$ is classically valid. A slightly more involved argument shows that also the existence property fails for classical logic.
} 
TheOREM 1.1. Let $\Gamma$ be a classical theory, i.e. a set of formulas not containing the symbols $\mathbb{\vee}$ and $\bar{\exists}$. Then for formulas $\varphi$ and $\psi$ the following holds

$$
\begin{aligned}
& \text { If } \Gamma \vDash \varphi \Downarrow \psi \text { then } \Gamma \vDash \varphi \text { or } \Gamma \vDash \psi \text {. } \\
& \text { If } \Gamma \vDash \bar{\exists} x . \varphi(x) \text { then } \Gamma \vDash \varphi(t) \text { for some term } t .
\end{aligned}
$$

That is, if a set of statements logically resolves an alternative question, then it supports one of the alternatives; and if a set of statements logically resolves an existential question, then there exists a term witnessing this resolution.

To prove this result, we develop several constructions to combine and transform information models. Some of them are inspired by operations on intuitionistic Kripke-frames (e.g., disjoint union), others are based on constructions typical of classical logic (e.g., models of terms, permutation models). Applying the developed theory, we obtain a purely semantic proof and further generalizations of Theorem 1.1. Moreover the introduced constructions seem interesting in their own right and might help in finding other meta-logical properties of InqBQ and its fragments, such as compactness and Löwenheim-Skolem type results. Summing up, this article initiates a modeltheoretic study of InqBQ and illustrates the results this approach could lead to.

Structure of the paper. Section 2 reviews inquisitive first-order logic InqBQ and the main properties that characterize it. In Section 3 we develop the toolkit of constructions used to prove the main results. In Section 4 we prove the general forms of the disjunction and existence properties. Section 5 presents further refinements of the disjunction property. Section 6 provides some concluding remarks and discusses open questions.

\section{Background: Preliminary Notions and the Logic InqBQ}

\subsection{Preliminaries}

Let $X, Y$ and $Z$ be sets and $\mathcal{A}=\left\langle A_{i} \mid i \in I\right\rangle$ a family of sets. We adopt the following notations: $X \times Y$ is the cartesian product of $X$ and $Y$, and $\pi_{1}^{X, Y}$, $\pi_{2}^{X, Y}$ (or simply $\pi_{1}$ and $\pi_{2}$ ) the corresponding projection maps defined as $\pi_{1}^{X, Y}(\langle x, y\rangle)=x$ and $\pi_{2}^{X, Y}(\langle x, y\rangle)=y ; \prod_{i \in I} A_{i}$ is the cartesian product of the family $\mathcal{A}$ and for each $i \in I$ we write $\pi_{i}^{\mathcal{A}}$ (or simply $\pi_{i}$ ) for the projection map defined as $\pi_{i}^{\mathcal{A}}\left(\left\langle a_{j} \mid j \in I\right\rangle\right)=a_{i} ; X \uplus Y:=(X \times\{0\}) \cup(Y \times\{1\})$ is the disjoint union of $X$ and $Y ; \biguplus_{i \in I} A_{i}:=\left\{\langle a, i\rangle \mid i \in I, a \in A_{i}\right\}$ is the disjoint union of the family $\left\langle A_{i} \mid i \in I\right\rangle$; given two functions with the same domain 
$f: Z \rightarrow X$ and $g: Z \rightarrow Y$, the function $\langle f, g\rangle: Z \rightarrow X \times Y$ is defined as $\langle f, g\rangle(z)=\langle f(z), g(z)\rangle$.

In the rest of the paper we will assume to have fixed a countable set of variables Var and a signature $\Sigma=\left\{f_{i}, R_{j}\right\}_{i \in I, j \in J}$, that is a set of symbols divided between function symbols and relation symbols, each of them with a corresponding arity specified by the function ar $: \Sigma \rightarrow \mathbb{N}$. In particular, we will call a function symbol of arity 0 a constant symbol.

With FO we indicate classical first order logic. We take the syntax of FO to be given by the following grammar:

$$
\alpha::=\perp\left|t_{1}=t_{2}\right| R_{j}(\bar{t})|\alpha \wedge \alpha| \alpha \rightarrow \alpha \mid \forall x . \alpha(x)
$$

where $t_{1}$ and $t_{2}$ are terms of $\Sigma$ and $\bar{t}$ is a sequence of terms of arity $\operatorname{ar} R_{j}$. Terms are defined in the usual way.

We will refer to formulas generated by this grammar as classical formulas. With abuse of notation, we will write $\alpha \in \mathrm{FO}$ to indicate that $\alpha$ is a classical formula.

A classical structure is a tuple

$$
M=\left\langle D, \mathbf{f}_{i}, \mathbf{R}_{j}, \sim\right\rangle_{i \in I, j \in J}
$$

where

- $D$ is a set of elements (the domain of the structure);

- $\mathbf{f}_{i}: D^{\operatorname{ar}\left(f_{i}\right)} \rightarrow D$ is the interpretation of the symbol $f_{i}$;

- $\mathbf{R}_{j} \subseteq D^{\mathrm{ar}\left(R_{j}\right)}$ is the interpretation of the symbol $R_{i}$;

- $\sim \subseteq D^{2}$ is a congruence with respect to the interpretation of function and relation symbols, ${ }^{2}$ called the identity of $M$.

We will refer to $D, \mathbf{f}_{i}, \mathbf{R}_{j}$ and $\sim$ also with $\operatorname{dom}(M), f_{i}^{M}, R_{j}^{M}$ and $\sim^{M}$ respectively.

A well known result is that, starting from a classical structure as defined above, is possible to obtain an isomorphic structure for which the relation $\sim$ is the real identity (i.e., the identity of the meta-theory). We indicate with $M / \sim$ the classical structure

$$
M / \sim=\left\langle D / \sim, \mathbf{f}_{i}^{\sim}, \mathbf{R}_{j}^{\sim},=\right\rangle_{i \in I, j \in J}
$$

\footnotetext{
${ }^{2} \mathrm{~A}$ congruence $\sim$ with respect to a relation $R$ is an equivalence relation such that $a R b \Longleftrightarrow \forall a^{\prime} \sim a . \forall b^{\prime} \sim b . a^{\prime} R b^{\prime}$. A congruence with respect to a function $f$ is a congruence with respect to the graph of $f$.
} 
where $\mathbf{f}_{i}^{\sim}$ and $\mathbf{R}_{j}^{\sim}$ are the interpretation induced by $\mathbf{f}_{i}$ and $\mathbf{R}_{j}$ on the quotient $D / \sim$, while $=$ is the real identity.

We define the skeleton $\operatorname{Sk}(M)$ of a classical structure $M$ as the tuple $\left\langle D, \mathbf{f}_{i}\right\rangle_{i \in I}$ consisting of the domain and function symbols' interpretations. More generally, we will refer to a tuple $S=\left\langle D, \mathbf{f}_{i}\right\rangle_{i \in I}$ as a skeleton.

Let $M$ be an arbitrary model and $g: \operatorname{Var} \rightarrow D$ an arbitrary valuation over $M$. The standard semantics of $\mathrm{FO}$, indicated by $\vDash^{\mathrm{FO}}$, is defined inductively by the following clauses:

$$
\begin{aligned}
& M \not \nvdash_{g}^{\mathrm{FO}} \perp \\
& M \vDash_{g}^{\mathrm{FO}} t_{1}=t_{2} \quad \Longleftrightarrow t_{1}^{g} \sim^{M} t_{2}^{g} \\
& M \models_{g}^{\mathrm{FO}} R\left(t_{1}, \ldots, t_{n}\right) \quad \Longleftrightarrow R^{M}\left(t_{1}^{g}, \ldots, t_{n}^{g}\right) \\
& M \vDash_{g}^{\mathrm{FO}} \psi_{1} \wedge \psi_{2} \quad \Longleftrightarrow M \vDash_{g}^{\mathrm{FO}} \psi_{1} \text { and } M \vDash_{g}^{\mathrm{FO}} \psi_{2} \\
& M \vDash_{g}^{\mathrm{FO}} \psi_{1} \rightarrow \psi_{2} \quad \Longleftrightarrow \text { if } M \vDash_{g}^{\mathrm{FO}} \psi_{1} \text { then } M \vDash_{g}^{\mathrm{FO}} \psi_{2} \\
& M \models_{g}^{\mathrm{FO}} \forall x . \psi \quad \Longleftrightarrow \text { For all } d \in D \text { it holds } M \vDash_{g[x \mapsto d]}^{\mathrm{FO}} \psi
\end{aligned}
$$

where $t^{g} \in D$ is the interpretation of term $t$ under the assignment $g$ computed by induction over the structure of $t$ in the usual way-and $g[x \mapsto$ $d]$ is the valuation over $M$ defined as

$$
g[x \mapsto d](y):= \begin{cases}d & \text { if } y=x \\ g(y) & \text { otherwise }\end{cases}
$$

We say that a formula $\varphi$ is true in $M$ under the assignment $g$ or that $M$ satisfy $\varphi$ under $g$ if $M \models_{g}^{\mathrm{FO}} \varphi$ holds. If $\varphi$ is a closed formula then the truth of $\varphi$ at $M$ is independent from the choice of $g$, so we will simply indicate $M \vDash^{\mathrm{FO}} \varphi$, omitting the assignment.

It is easy to verify the following relation between structures $M$ and $M / \sim$ for an arbitrary assignment $g$ over $M$ :

$$
M \vDash_{g}^{\mathrm{FO}} \varphi \Longleftrightarrow M / \sim \vDash_{g^{\sim}}^{\mathrm{FO}} \varphi
$$

where $g^{\sim}(x)=[g(x)]_{\sim}$, the equivalence class of $g(x)$ under $\sim$.

\subsection{Syntax of InqBQ}

In the next subsections we quickly present the logic InqBQ and the properties that characterize this logic. For a more thorough treatment of the subject we refer to [3]. Here we start by presenting the syntax of the logic.

Definition 2.1. (Syntax of InqBQ) The syntax of the logic InqBQ is generated by the following grammar: 


$$
\varphi::=\perp\left|t_{1}=t_{2}\right| R(\bar{t})|\varphi \wedge \varphi| \varphi \backslash \varphi|\varphi \rightarrow \varphi| \forall x . \varphi \mid \bar{\exists} x . \varphi
$$

where $t_{1}$ and $t_{2}$ are terms of $\Sigma$ and $\bar{t}$ is a sequence of terms of arity $\operatorname{ar}(R)$.

The syntax here introduced is that of FO with in addition the two new operators $\mathbb{V}$ (inquisitive disjunction) and $\bar{\exists}$ (inquisitive existential quantifier). To enrich the language and point out the differences with the corresponding classical operators, we introduce the usual shorthands

$$
\neg \varphi:=\varphi \rightarrow \perp \quad \varphi \vee \psi:=\neg(\neg \varphi \wedge \neg \psi) \quad \exists x . \varphi:=\neg \forall x . \neg \varphi
$$

In accordance with the terminology previously adopted, we will call a formula classical if it does not contain the symbols $\mathbb{\vee}$ and $\bar{\exists}$. In particular, given two classical formulas $\varphi$ and $\psi$, also $\neg \varphi, \varphi \vee \psi$ and $\exists x . \varphi$ are classical formulas.

Henceforth we will indicate with the symbols $\alpha, \beta, \gamma, \ldots$ classical formulas, while we will use the symbols $\varphi, \chi, \psi, \ldots$ to indicate generic formulas.

\subsection{Models of InqBQ}

The logic InqBQ aims to capture the logical relations between questions through an information based approach, as previously mentioned in Section 1. To formally represent a body of information, a new mathematical structure is hereby introduced.

Definition 2.2. (Information model) An information model $\mathcal{M}$ is a tuple $\left\langle M_{w} \mid w \in W\right\rangle$ where $W$ is a set - called the set of worlds of $\mathcal{M}$ and denoted by $\operatorname{wrd}(\mathcal{M})$ - and the $M_{w}$ are classical structures sharing the same skeleton.

We will denote with $\operatorname{Sk}(\mathcal{M})$ the shared skeleton of the structures; with $\operatorname{dom}(\mathcal{M})$ and $f_{i}^{\mathcal{M}}$ the common domain and the common interpretation of the function symbol $f_{i}$; we sometimes abbreviate the interpretation $R_{j}^{M_{w}}$ of the symbol $R_{j}$ in the model $M_{w}$ as $R_{j}^{w}$, and the interpretation $\sim^{M_{w}}$ of the identity in $M_{w}$ as $\sim^{w}$.

Notice that to define an information model it suffices to specify separately $\operatorname{wrd}(\mathcal{M}), \operatorname{Sk}(\mathcal{M})$ and, for every $w \in \operatorname{wrd}(\mathcal{M}), R_{j}^{w}$ and $\sim^{w}$. To ensure that an information model with this structure exists it is necessary and sufficient to check that for every world $w \in \operatorname{wrd}(\mathcal{M})$ the relation $\sim^{w}$ is a congruence with respect to the interpretation of the symbols.

Intuitively, information models act as a context in which we can represent information using the following approach: an information state is encoded by the set of worlds compatible with it. 


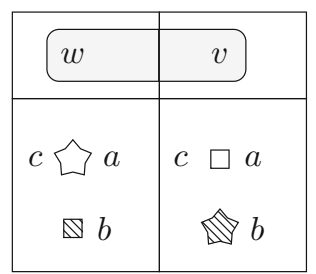

Figure 1. A graphical representation of an information model in the signature $\Sigma=\{c, P\}$

Definition 2.3. (Information state) Given a model $\mathcal{M}$, we will refer to a subset $s \subseteq \operatorname{wrd}(\mathcal{M})$ as an information state or info state. We say that $t$ is an enhancement of $s$ if $t \subseteq s$.

We define the submodel of $\mathcal{M}$ relative to $s$ as $\mathcal{M}_{s}=\left\langle M_{w} \mid w \in s\right\rangle$.

EXAMPLE 2.4. A graphical representation of an information model $\mathcal{M}$ is depicted in Figure 1. The signature considered is $\Sigma=\{c, P\}$, consisting of a constant symbol $c$ and a unary predicate $P$. $\mathcal{M}$ is defined by the following clauses.

- The set of worlds of $\mathcal{M}$ is $\{w, v\}$. In Figure 1 , the column with label $w$ represents the classical structure $M_{w}$, and similarly for $v$.

- $\operatorname{dom}(\mathcal{M})=\{a, b\}$. In Figure 1, we depict one copy of the domain for every classical structure.

- $c^{\mathcal{M}}=a$. In Figure 1, it is indicated by placing the symbol $c$ near $a$. Notice that by definition the interpretation of $c$ has to be the same for every world.

- For every column, the elements in the extension of $P$ are represented by star-shaped nodes, while the others are represented by square-shaped nodes. In Figure 1 we have $P^{w}(a), P^{v}(b)$ but not $P^{w}(b), P^{v}(a)$.

- For every column, identity is represented by the pattern of the nodes. Two elements in the column labelled $w$ are related by the identity $\sim^{w}$ of $M_{w}$ if and only if they have the same pattern; similarly for $v$. In Figure 1 we have $a \chi^{w} b$ and $a \chi^{v} b$.

Finally, the grey rectangle represents the information state $s=\{w, v\} . s$ represents the following informational scenario: it is known that there are two distinct individuals and that exactly one of these individuals has property $P$. It is not known, however, which of the two individuals have property $P$. 


\begin{tabular}{|c|c|c|}
\hline$w_{1}$ & $w_{2}$ & $w_{3}$ \\
\hline $\begin{array}{ccccccc} & & -2 & -1 & 0 & 1 & 2 \\
\cdots & \mathbb{N} & \mathbb{N} & \mathbb{Q} & \mathbb{Q} & \mathbb{Q} & \cdots\end{array}$ & 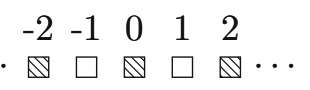 & 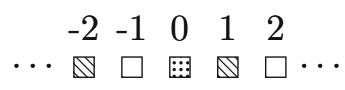 \\
\hline
\end{tabular}

Figure 2. A graphical representation of the first-order structures associated in $\mathbb{Z}_{*}$ to $w_{1}, w_{2}$ and $w_{3}$ respectively. The same conventions as Figure 1 are adopted. The classical structures associated with two distinct worlds are distinguished only by the identity relation; for example, at world $w_{2}$ it holds $-2 \sim^{w_{2}} 2$ because $-2 \equiv 2 \bmod 2$, while at world $w_{3}$ we have $-2 \not{ }^{w_{3}} 2$ because $-2 \not \equiv 2 \bmod 3$

Example 2.5. (A model representing all finite cyclic groups) Consider the signature $\{0,+\}$ and the set of worlds $W=\mathbb{N} \backslash\{0\}$ (we will write $w_{n}$ instead of $n$ to point out the number is used as a world). Consider the tuple $\mathbb{Z}_{*}=\left\langle\mathbb{Z} / n \mathbb{Z} \mid w_{n} \in W\right\rangle$ where $\mathbb{Z} / n \mathbb{Z}$ indicates the group $\mathbb{Z}$ with identity interpreted as the relation $a \sim^{n} b$ iff $a-b \equiv 0 \bmod n$. A graphical representation of this model is given in Figure 2.

\subsection{Semantics of InqBQ}

Now we have all the ingredients needed to introduce the semantics for the logic InqBQ.

Definition 2.6. (Semantics of InqBQ) Let $\mathcal{M}$ be an information model, $s \subseteq \operatorname{wrd}(\mathcal{M})$ an info state and $g: \operatorname{Var} \rightarrow \operatorname{dom}(\mathcal{M})$ an assignment. We define the support relation $\vDash$ over formulas of InqBQ by the following inductive clauses:

$\mathcal{M}, s \vDash_{g} \perp$

$\Longleftrightarrow s=\emptyset$

$\mathcal{M}, s \vDash_{g} t_{1}=t_{2}$

$\Longleftrightarrow$ For all $w \in s$ it holds $t_{1}^{g} \sim^{M_{w}} t_{2}^{g}$

$\mathcal{M}, s \vDash_{g} R\left(t_{1}, \ldots, t_{n}\right)$

$\Longleftrightarrow$ For all $w \in s$ it holds $R^{M_{w}}\left(t_{1}^{g}, \ldots, t_{n}^{g}\right)$

$\mathcal{M}, s \vDash_{g} \psi_{1} \wedge \psi_{2}$

$\Longleftrightarrow \mathcal{M}, s \vDash_{g} \psi_{1}$ and $\mathcal{M}, s \vDash_{g} \psi_{2}$

$\mathcal{M}, s \vDash_{g} \psi_{1} \mathbb{V} \psi_{2}$

$\Longleftrightarrow \mathcal{M}, s \vDash_{g} \psi_{1}$ or $\mathcal{M}, s \vDash_{g} \psi_{2}$

$\mathcal{M}, s \vDash_{g} \psi_{1} \rightarrow \psi_{2}$

$\Longleftrightarrow$ For all $t \subseteq s$, if $\mathcal{M}, t \vDash_{g} \psi_{1}$ then $\mathcal{M}, t \vDash_{g} \psi_{2}$

$\mathcal{M}, s \vDash_{g} \forall x . \psi$

$\Longleftrightarrow$ For all $d \in \operatorname{dom}(\mathcal{M})$ it holds $\mathcal{M}, s \vDash_{g[x \mapsto d]} \psi$

$\mathcal{M}, s \vDash_{g} \bar{\exists} x . \psi$

$\Longleftrightarrow$ There is $d \in \operatorname{dom}(\mathcal{M})$ such that $\mathcal{M}, s \vDash_{g[x \mapsto d]} \psi$

Given $\Phi$ a set of formulas, with the notation $\mathcal{M}, s \vDash_{g} \Phi$ we indicate that for every formula $\varphi \in \Phi$ it holds $\mathcal{M}, s \vDash_{g} \varphi$. 
In case $s=\operatorname{wrd}(\mathcal{M})$ we will simply write $\mathcal{M} \vDash_{g} \varphi$ and $\mathcal{M} \vDash_{g} \Phi$, omitting the info state.

We define the entailment relation of InqBQ as follows: given a theory $\Phi$ and a formula $\psi$, we indicate with $\Phi \vDash \psi$ that for every $\mathcal{M}, s$ and $g$ such that $\mathcal{M}, s \vDash_{g} \Phi$ it also holds $\mathcal{M}, s \vDash_{g} \psi$. In particular, we indicate with $\varphi \equiv \psi$ that $\varphi \vDash \psi$ and $\psi \vDash \varphi$.

The intuition behind this semantics is the one mentioned in Section 1: an information state supports a statement if the statement is implied by the information state; and an information state supports a question if the issue raised by the question is resolved by the information state. So for example, according to the semantics, the formula $P(c)$ representing the statement " $c$ has property $P$ " is supported by the info state $s$ if and only if every classical structure $M_{w}$ associated with a world $w \in s$ satisfies $P(c)$. And the formula $\exists x . P(x)$ representing the question "What is an element with property $P$ ?" is supported by $s$ if and only if we can identify an element $a$ such that $P(a)$ is satisfied for every $M_{w}$ with $w \in s$.

Two characteristic properties of this semantics - which can be easily proved by structural induction - are the following:

Lemma 2.7. For every formula $\varphi$ of the logic

Empty set property $\mathcal{M}, \emptyset \vDash_{g} \varphi$.

Persistency If $\mathcal{M}, s \vDash_{g} \varphi$ and $u \subseteq s$, then $\mathcal{M}, u \vDash_{g} \varphi$.

These two properties have a clear conceptual interpretation: the incoherent information supports everything (empty set) and every enhancement of an information that supports a sentence still supports the same sentence (persistency).

The support semantics here introduced strongly resembles the forcing relation for intuitionistic Kripke models (see for example [6]). In fact there is a tight relation between the two semantics, which was already presented in [2]: given an information model $\mathcal{M}$ we can define a corresponding intuitionistic Kripke model $\mathcal{M}^{\prime}$ forcing all and only the formulas supported by $\mathcal{M}$-modulo interpreting $\mathbb{V}$ and $\bar{\exists}$ as intuitionistic disjunction and intuitionistic existential quantifier respectively. A more detailed study of the relation between the two logics in connection with the results introduced in this paper is left for future work.

Using Definition 2.6 and Lemma 2.7, we can also compute support conditions for the shorthands $\neg \varphi, \varphi \vee \psi$ and $\exists x . \varphi$ : 


$$
\begin{aligned}
\mathcal{M}, s \vDash_{g} \neg \varphi & \Longleftrightarrow \text { For all } w \in s \text { it holds } \mathcal{M},\{w\} \vDash_{g} \varphi \\
\mathcal{M}, s \vDash_{g} \varphi \vee \psi & \Longleftrightarrow \text { For all } w \in s \text { it holds: } \mathcal{M},\{w\} \vDash_{g} \varphi \text { or } \mathcal{M},\{w\} \vDash_{g} \psi \\
\mathcal{M}, s \vDash_{g} \exists x . \varphi & \Longleftrightarrow \text { For all } w \in s \text { there exists } d_{w} \in \operatorname{dom}(\mathcal{M}) \\
& \text { such that } \mathcal{M}, s \vDash_{g\left[x \mapsto d_{w}\right]} \varphi
\end{aligned}
$$

Notice that the semantical clauses for $\bar{\exists}$ and for its classical counterpart $\exists$ are quite different. For example, the formula $\exists x . P(x)$, representing the sentence "There is an element with property $P$ ", is supported iff for every world contained in the current information state there is an element-dependent on the world - with property $P$. We just need to know that an element with property exists, even if we cannot pinpoint which. On the other hand, the formula $\bar{\exists} x \cdot P(x)$, representing the question "Is there an element with property $P$ ?", has a more demanding condition: this formula is supported iff there exists a determinate element with property $P$ for every world contained in the current information state - that is, we have a definite answer to the question. Going back to Figure 1, we can show that the two formulas are indeed not equivalent: the highlighted information state $s$ satisfies $\exists x . P(x)$, but not $\bar{\exists} x . P(x)$.

Now that we have defined the entailment relation of the logic we can also see how the relations of determinacy, presupposition and resolution are formalized as a facet of the entailment relation.

ExAmple 2.8. Consider the sentences (a)-(d) presented in Section 1. It is easy to prove that the following entailments between formulas representing the sentences hold.

$$
\begin{aligned}
\bar{\exists} x .(P(x) \wedge Q(x)) & \models \bar{\exists} x \cdot P(x) & & \text { (d) determines (c) } \\
\bar{\exists} x . P(x) & \models \exists x . P(x) & & \text { (c) presupposes (b) } \\
P(c) & \models \bar{\exists} x . P(x) & & \text { (a) resolves (c) }
\end{aligned}
$$

For a more exhaustive treatment of the connection between entailment and the relations introduced above we refer to [4].

\subsection{Statements and Truth-Conditionality}

In this subsection we present some of the main properties characterizing InqBQ that will be used in later sections. We start with a simple remark that shows that the semantics introduced is indeed a generalization of the semantics of FO.

REMARK 2.9. It is easy to prove that for a classical formula $\alpha$ it holds

$$
\mathcal{M}, s \vDash_{g} \alpha \Longleftrightarrow \forall w \in s . M_{w} \vDash_{g}^{\mathrm{FO}} \alpha
$$


In particular, this means that we can recover the semantics of FO by simply restricting the syntax to classical formulas and the semantics to single-world information models (i.e., models for which $\operatorname{wrd}(\mathcal{M})$ is a singleton).

This result has also a clear conceptual interpretation: a sentence is supported by an information state if and only if in every scenario (world) compatible with the information state the sentence is true. That is, a classical formula is completely determined by its truth-conditions.

This remark naturally leads to the following definition.

Definition 2.10. A formula $\varphi$ is called truth-conditional if for every model $\mathcal{M}$, info state $s$ and assignment $g$ it holds

$$
\mathcal{M}, s \vDash_{g} \varphi \Longleftrightarrow \forall w \in s . \mathcal{M},\{w\} \vDash_{g} \varphi
$$

The formulas $\neg \varphi, \varphi \vee \psi$ and $\exists x . \varphi$ are always truth-conditional (compare with the semantical clauses given in Section 2.4). From this fact and from Remark 2.9 we can easily infer the equivalences

$$
\neg(\neg \varphi \vee \neg \psi) \equiv \neg \neg \varphi \wedge \neg \neg \psi \quad \neg \bar{\exists} x . \neg \varphi \equiv \forall x . \neg \neg \varphi
$$

The next Lemma follows easily from Remark 2.9.

\section{LEMMA 2.11. Every classical formula $\alpha$ is truth-conditional.}

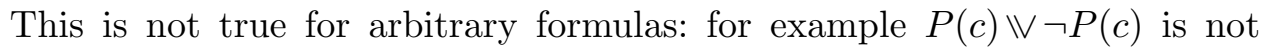
truth-conditional.

As we recalled in Section 1 and in line with the interpretation above, FO is a logic meant to represent statements, that is exactly those sentences completely determined by their truth-conditions. The definition of truthconditional formula given above expresses formally the same property: the support conditions of a truth-conditional formula $\varphi$ are completely determined by the worlds in which the formula is true. At this point a question naturally arises: in InqBQ, are there statements not represented by classical formulas? The negative answer is given by the following Theorem. A proof can be found in [3] $\S 4$.

Theorem 2.12. (Truth conditionality and classical formulas) For every formula $\varphi, \varphi$ is truth conditional if and only if there exists a classical formula such that $\varphi \equiv \alpha$.

We conclude this subsection with another fundamental result about InqBQ: combining Lemma 2.7 with Lemma 2.11 we obtain that the entailment relation of InqBQ - like its semantics - is a generalization of its classical counterpart. 
TheOREM 2.13. For every set of classical formulas $\Gamma \cup\{\alpha\}$ it holds

$$
\Gamma \vDash \alpha \Longleftrightarrow \Gamma \vDash^{\mathrm{FO}} \alpha
$$

that is, $\vDash$ is a conservative extension of $\models^{\mathrm{F} 0}$.

\section{Model Constructions}

We now present several constructions to transform and combine information models. The techniques introduced here will be used in the next sections to study various aspects of the logic InqBQ.

We start with some technical results that will be useful throughout the paper.

Definition 3.1. Let $\mathcal{M}$ and $\mathcal{N}$ be two information models. Let $s$ and $t$ be info states of $\mathcal{M}$ and $\mathcal{N}$ respectively. Let $G: \operatorname{dom}(\mathcal{M}) \rightarrow \operatorname{dom}(\mathcal{N})$ be a surjective function. We say that $s$ and $t$ are quasi-isomorphic under $G$ if

$$
\begin{aligned}
& \forall w \in s . \exists w^{\prime} \in t . M_{w} \cong_{G} N_{w^{\prime}} \\
& \forall w^{\prime} \in t . \exists w \in s . M_{w} \cong_{G} N_{w^{\prime}}
\end{aligned}
$$

where $M_{w} \cong_{G} N_{w^{\prime}}$ indicates that $G$ is an isomorphism of FO structures between $M_{w}$ and $N_{w^{\prime}}$.

Lemma 3.2. Let $\mathcal{M}, \mathcal{N}, G, s$ and $t$ be as in the definition above and suppose that $s$ and $t$ are quasi-isomorphic under $G$. Let $g$ be an assignment on $\mathcal{M}$. Then for every formula $\varphi$ it holds

$$
\mathcal{M}, s \vDash_{g} \varphi \Longleftrightarrow \mathcal{N}, t \vDash_{G \circ g} \varphi
$$

The proof, omitted here, consists of a simple structural induction.

Definition 3.3. Let $\mathcal{M}$ and $\mathcal{N}$ be two models and consider two maps $F: \operatorname{wrd}(\mathcal{M}) \rightarrow \operatorname{wrd}(\mathcal{N})$ and $G: \operatorname{dom}(\mathcal{M}) \rightarrow \operatorname{dom}(\mathcal{N})$. We call the pair $\langle F, G\rangle$ an $\mathcal{I}$-morphism from $\mathcal{M}$ to $\mathcal{N}$ if:

1. $G$ is surjective;

2. $G$ commutes with functions:

$$
G\left(f^{\mathcal{M}}\left(d_{1}, \ldots, d_{n}\right)\right)=f^{\mathcal{N}}\left(G\left(d_{1}\right), \ldots, G\left(d_{n}\right)\right)
$$

3. $\langle F, G\rangle$ respects relations: for every $w \in \operatorname{wrd}(\mathcal{M}), d_{1}, \ldots, d_{n} \in \operatorname{dom}(\mathcal{M})$

$$
R^{w}\left(d_{1}, \ldots, d_{n}\right) \text { iff } R^{F(w)}\left(G\left(d_{1}\right), \ldots, G\left(d_{n}\right)\right)
$$



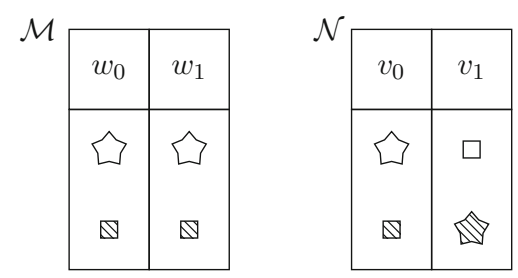

Figure 3. Given the models $\mathcal{M}$ and $\mathcal{N}$ depicted in figure (the extension of property $P$ is indicated with the star), the map $F\left(w_{i}\right)=v_{i}$ induces an isomorphism between the classical structures $M_{w_{i}}$ and $N_{v_{i}}$, but the two models are not elementarily equivalent. For example, the formula $\bar{\exists} x . P(x)$ distinguishes the two models: in $\mathcal{M}$ the information we have is enough to ensure that $P$ holds at the white element; in $\mathcal{N}$ we know that an element has property $P$, but we do not know if it is the white one or the striped one

4. $\langle F, G\rangle$ respects identity: for every $w \in \operatorname{wrd}(\mathcal{M}), d_{1}, \ldots, d_{n} \in \operatorname{dom}(\mathcal{M})$

$$
d_{1} \sim^{w} d_{2} \text { iff } G\left(d_{1}\right) \sim^{F(w)} G\left(d_{2}\right)
$$

Conditions (3) and (4) ensure that $G$ induces for every $w \in \operatorname{wrd}(\mathcal{M})$ a map between the models $M_{w} / \sim^{w}$ and $N_{F(w)} / \sim^{F(w)}$. Adding conditions (1) and (2) also ensures that the induced map is an isomorphism of classical structures. This is formalized by the following Lemma.

In general, having a bijective map $F: \operatorname{wrd}(\mathcal{M}) \rightarrow \operatorname{wrd}(\mathcal{N})$ such that $M_{w} / \sim^{w}$ and $N_{F(w)} / \sim^{F(w)}$ are isomorphic is not enough to preserve supported formulas, as shown in Figure 3.

Lemma 3.4. Let $\mathcal{M}$ and $\mathcal{N}$ be two models and suppose there exists an $\mathcal{I}$ morphism $\langle F, G\rangle$ from $\mathcal{M}$ to $\mathcal{N}$. Then for every $s \subseteq \operatorname{wrd}(\mathcal{M})$, for every assignment $h: \operatorname{Var} \rightarrow \operatorname{dom}(\mathcal{M})$ and for every formula $\varphi$

$$
\mathcal{M}, s \vDash_{h} \varphi \Longleftrightarrow \mathcal{N}, F(s) \vDash_{G \circ h} \varphi
$$

Proof. The info states $s$ and $F(s)$ are quasi-isomorphic, so by Lemma 3.2 we then obtain the result.

\subsection{Extending the Skeleton}

Here we present several ways to modify the skeleton of a given model without changing its logical properties.

The first and most intuitive way to modify the skeleton is to add copies of the elements. 


\begin{tabular}{|c|c|}
\hline$v_{0}$ & $v_{1}$ \\
\hline$\langle a, 0\rangle\langle a, 1\rangle\langle a, 2\rangle$ & $\langle a, 0\rangle\langle a, 1\rangle\langle a, 2\rangle$ \\
\hline$c \hat{\Delta} \Omega \cdots$ & $c \square \quad \square \quad \square \cdots$ \\
\hline$\langle b, 0\rangle\langle b, 1\rangle\langle b, 2\rangle$ & $\langle b, 0\rangle\langle b, 1\rangle\langle b, 2\rangle$ \\
\hline $\mathbb{\nabla} \quad \mathbb{Q} \quad \mathbb{Q} \cdots$ & $\mathbb{N}$ \\
\hline
\end{tabular}

Figure 4 . In the picture, the model $\mathcal{M}_{\omega}$ obtained from the model $\mathcal{M}$ of Figure 1 is depicted. The new domain is obtained by adding a countable amount of copies of each element. For example, the elements in the gray box are the copies of the element $a$ and are $\sim$-equivalent at every world of the model

Definition 3.5. Given a model $\mathcal{M}=\left\langle M_{w} \mid w \in W\right\rangle$ and $\delta$ an ordinal, we define the model $\mathcal{M}_{\delta}=\left\langle M_{w}^{\delta} \mid w \in W^{\delta}\right\rangle$ by the following clauses:

- $W^{\delta}=W$;

- $\operatorname{dom}\left(\mathcal{M}_{\delta}\right)=\operatorname{dom}(\mathcal{M}) \times \delta$;

- $f^{\mathcal{M}_{\delta}}\left(\left\langle d_{1}, \gamma_{1}\right\rangle, \ldots,\left\langle d_{n}, \gamma_{n}\right\rangle\right)=\left\langle f^{\mathcal{M}}\left(d_{1}, \ldots, d_{n}\right), 0\right\rangle$;

- $R^{M_{w}^{\delta}}\left(\left\langle d_{1}, \gamma_{1}\right\rangle, \ldots,\left\langle d_{n}, \gamma_{n}\right\rangle\right)$ iff $R^{M_{w}}\left(d_{1}, \ldots, d_{n}\right)$;

- $\left\langle d_{1}, \gamma_{1}\right\rangle \sim^{M_{w}^{\delta}}\left\langle d_{2}, \gamma_{2}\right\rangle$ iff $d_{1} \sim^{M_{w}} d_{2}$.

The following lemma formalizes the idea that we only added redundant information.

Lemma 3.6. Let $s \subseteq \operatorname{wrd}(\mathcal{M})$ be an info state and $g: \operatorname{Var} \rightarrow \operatorname{dom}(\mathcal{M}) \times \delta$ an assignment. Then for every formula $\varphi$

$$
\mathcal{M}_{\delta}, s \vDash_{g} \varphi \Longleftrightarrow \mathcal{M}, s \vDash_{\pi_{1} \circ g} \varphi
$$

Proof. The functions id $: \operatorname{wrd}\left(\mathcal{M}_{\delta}\right) \rightarrow \operatorname{wrd}(\mathcal{M})$ and $\pi_{1}: \operatorname{dom}(\mathcal{M}) \times \delta \rightarrow$ $\operatorname{dom}(\mathcal{M})$ respect the hypothesis of Lemma 3.4, thus the result follows.

The construction above can be thought of as a product between a model and the set $\delta$, and it is easy to generalize this to an arbitrary set.

A slightly more challenging task is to define a product between a model and a skeleton. The idea is to encode in a single structure both the components of the model (domain, functions, relations, identity) and the components of the skeleton (domain, functions), in a way that let us recover them easily. 


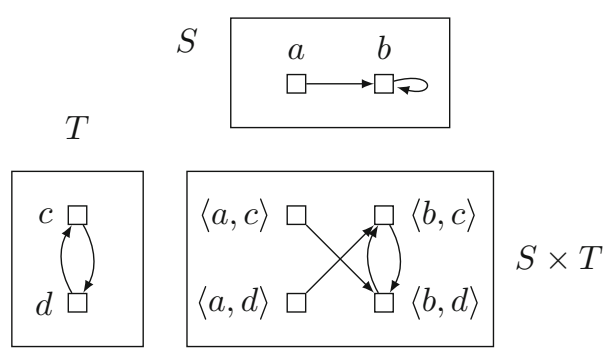

Figure 5. A simple example of the product of two skeletons in the signature $\Sigma=\{f\}$, for $f$ a function symbol of arity one. In particular, the arrows represent the interpretation of $f$ (the target is the image of the source)

Given two skeletons $S$ and $T$ we can naturally define their product $S \times T$ by the following clauses:

- $\operatorname{dom}(S \times T)=\operatorname{dom}(S) \times \operatorname{dom}(T)$;

- $f^{S \times T}\left(\left\langle d_{1}, e_{1}\right\rangle, \ldots,\left\langle d_{n}, e_{n}\right\rangle\right)=\left\langle f^{S}\left(d_{1}, \ldots, d_{n}\right), f^{T}\left(e_{1}, \ldots, e_{n}\right)\right\rangle$.

It is immediate to generalize the notion of product to an arbitrary family of skeletons $\left\langle S_{i} \mid i \in I\right\rangle$ : we indicate with $\prod_{i \in I} S_{i}$ the product of the family. See Figure 5 for a simple example of product of skeletons.

Building up on this, we define the following construction:

Definition 3.7. (Product of skeletons) Given a model $\mathcal{M}=\left\langle M_{w} \mid w \in W\right\rangle$ and a skeleton $S$, we define the model $\mathcal{M} \rtimes S=\left\langle M_{w}^{\rtimes} \mid w \in W^{\rtimes}\right\rangle$, the product of $\mathcal{M}$ and $S$, by the following clauses

- $\operatorname{Sk}(\mathcal{M} \rtimes S)=\operatorname{Sk}(\mathcal{M}) \times S$;

- $W^{\rtimes}=W$;

- $R^{M_{w}^{\rtimes}}\left(\left\langle d_{1}, e_{1}\right\rangle, \ldots,\left\langle d_{n}, e_{n}\right\rangle\right)$ iff $R^{M_{w}}\left(d_{1}, \ldots, d_{n}\right)$;

- $\left\langle d_{1}, e_{1}\right\rangle \sim^{\mathcal{M}} \underset{w}{\rtimes}\left\langle d_{2}, e_{2}\right\rangle$ iff $d_{1} \sim^{M_{w}} d_{2}$.

It is easy to show that $\mathcal{M} \rtimes S$ is a well-defined model. With the same proof as in Lemma 3.6 we obtain the following.

Lemma 3.8. Let $s \subseteq \operatorname{wrd}(\mathcal{M})$ be an info state and $g: \operatorname{Var} \rightarrow \operatorname{dom}(\mathcal{M}) \times$ $\operatorname{dom}(S)$ an assignment. Then for every formula $\varphi$

$$
\mathcal{M} \rtimes S, s \vDash_{g} \varphi \Longleftrightarrow \mathcal{M}, s \vDash_{\pi_{1} \circ g} \varphi
$$

We can generalize Definition 3.7 to the product between a model $\mathcal{M}$ and an arbitrary family of skeletons $\left\langle S_{i} \mid i \in I\right\rangle$. This way we obtain a model whose 
skeleton is $\operatorname{Sk}(\mathcal{M}) \times \prod_{i \in I} S_{i}$ and for which a Lemma analogous to Lemma 3.8 holds. The details are left to the reader.

We focus now on a different approach to modify the skeleton, namely by extending the domain to an algebra of terms in a similar fashion to Hintikka's model of terms (as presented in [8], Section 2.3). This construction makes the structure of a model less rigid, while preserving the support of InqBQ formulas.

Given a set $A$, fix a family $\{\underline{a} \mid a \in A\}$ of fresh constants not present in $\Sigma$. We define the algebra of $\Sigma$-terms on $A$ as

$$
\Sigma[A]=\left\{t\left(\underline{a_{1}}, \ldots, \underline{a_{n}}\right) \mid t\left(x_{1}, \ldots, x_{n}\right) \text { term of } \Sigma \text { and } a_{1}, \ldots, a_{n} \in A\right\}
$$

We will often take $A=\operatorname{dom}(\mathcal{M})$ for some model $\mathcal{M}$. In this case, if $e \in$ $\operatorname{dom}(\mathcal{M})$ is the interpretation of a constant term $c$, in $\Sigma[\operatorname{dom}(\mathcal{M})]$ we have both $\underline{e}$ and $c$ as distinct elements.

We can easily impose a structure of skeleton on this set: we define $\mathbf{T} A$ by the following clauses:

- $\operatorname{dom}(\mathbf{T} A)=\Sigma[A]$

- $f^{\mathbf{T} A}\left(t_{1}, \ldots, t_{n}\right)=f\left(t_{1}, \ldots, t_{n}\right)$ (notice that $f^{\mathbf{T} A}$ is a function while $f$ is a formal symbol).

If $A=\operatorname{dom}(S)$ is the domain of a skeleton $S$, we can define a natural projection $\tau: \Sigma[\operatorname{dom}(S)] \rightarrow \operatorname{dom}(S)$ by the following clauses:

$$
\tau(\underline{a})=a \quad \tau\left(f\left(t_{1}, \ldots, t_{n}\right)\right)=f^{S}\left(\tau\left(t_{1}\right), \ldots, \tau\left(t_{n}\right)\right)
$$

These considerations lead us to the following construction:

Definition 3.9. (Term model) Given a model $\mathcal{M}=\left\langle M_{w} \mid w \in W\right\rangle$, we define $\mathbf{T} \mathcal{M}=\left\langle M_{w}^{\mathbf{T}} \mid w \in W^{\mathbf{T}}\right\rangle$, the term model of $\mathcal{M}$, by the following clauses

- $W^{\mathbf{T}}=W$;

- $\operatorname{Sk}(\mathbf{T} \mathcal{M})=\mathbf{T d o m}(\mathcal{M})$;

- $R^{M_{w}^{\mathbf{T}}}\left(t_{1}, \ldots, t_{n}\right)$ iff $R^{M_{w}}\left(\tau\left(t_{1}\right), \ldots, \tau\left(t_{n}\right)\right)$;

- $t_{1} \sim^{M_{w}^{\mathrm{T}}} t_{2}$ iff $\tau\left(t_{1}\right) \sim^{M_{w}} \tau\left(t_{2}\right)$.

We can interpret $\sim^{\mathbf{T M}}$ as an extension of $\sim^{\mathcal{M}}$. In fact given $d, d^{\prime} \in \operatorname{dom}(\mathcal{M})$, the congruence condition for $\underline{d}$ and $\underline{d^{\prime}}$ reduces to $\underline{d} \sim^{M_{w}^{\mathrm{T}}} \underline{d^{\prime}}$ iff $d \sim^{M_{w}} d^{\prime}$.

A graphical representation of the term model of a simple model is given in Figure 6. 

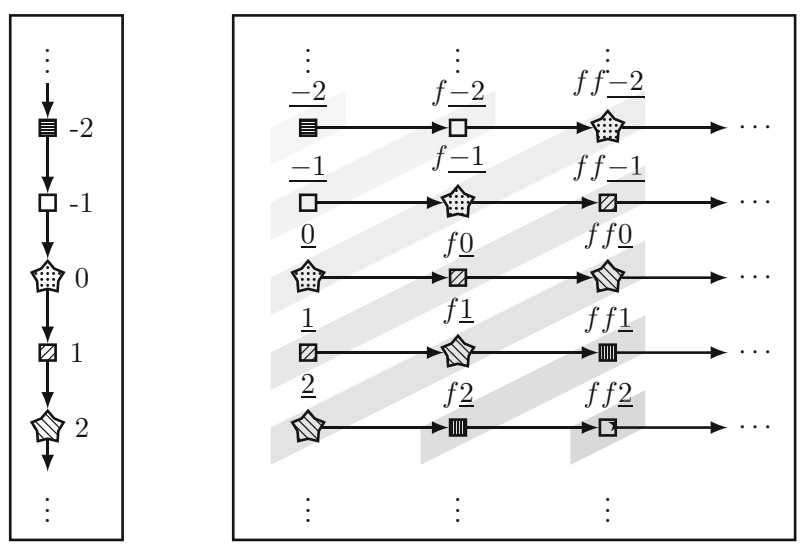

Figure 6. An example of term model of a single-world model (i.e., a model such that $|\operatorname{wrd}(\mathcal{M})|=1)$ in the signature $\Sigma=\left\{f^{(1)}\right\}$. The model $\mathcal{M}$ (on the left) represents the set $\mathbb{Z}$ where $f$ (depicted by the arrows) is interpreted as the usual successor function and identity (represented by patterns) is interpreted as the real identity. The model $\mathbf{T} \mathcal{M}$ (on the right) contains all the terms of the extended signature $\Sigma(\mathbb{Z})$ and interprets the function $f$ as the formal term combinator (i.e., $f^{\mathbf{T M}}(t)=f(t)$ ). The equivalence classes of the relation $\sim^{\mathbf{T M}}$ have been highlighted in different shades of gray

Using the projection $\tau$, we can show that yet again we obtained a model carrying the same information as $\mathcal{M}$.

TheOREm 3.10. Let $s \subseteq \operatorname{wrd}(\mathcal{M})$ and $g: \operatorname{Var} \rightarrow \Sigma[\operatorname{dom}(\mathcal{M})]$. Then for every formula $\varphi$

$$
\mathbf{T} \mathcal{M}, s \vDash_{g} \varphi \Longleftrightarrow \mathcal{M}, s \vDash_{\tau \circ g} \varphi
$$

Proof. The functions id $: \operatorname{wrd}(\mathbf{T} \mathcal{M}) \rightarrow \operatorname{wrd}(\mathcal{M})$ and $\tau$ respect the hypothesis of Lemma 3.4, thus the result follows.

\subsection{Combining Models}

In this section we present two techniques to combine different models.

The first construction gives us a natural way to combine two models into a new one, with the caveat that they must have the same skeleton. Under this hypothesis, we can obtain a structure encoding the information carried by the two models and from which we can extract the information in a natural way. 

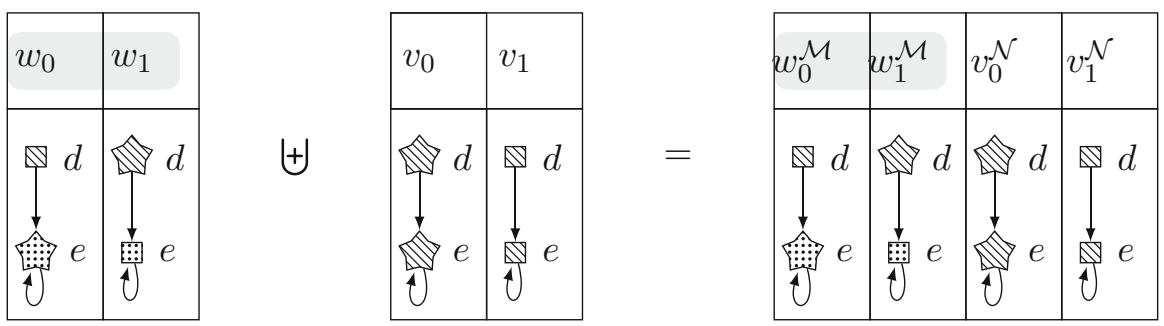

Figure 7. The disjoint union of the models $\mathcal{M}$ (first model) and $\mathcal{N}$ (second model). In the picture, the info state $s$ of $\mathcal{M}$ (highlighted in gray in the first model) naturally corresponds to the state $s^{\mathcal{M}}$ of $\mathcal{M} \uplus \mathcal{N}$ (highlighted in gray in the third model)

Definition 3.11. (Disjoint union) Let $\mathcal{M}=\left\langle M_{w} \mid w \in W\right\rangle$ and $\mathcal{N}$ be two models with $\operatorname{Sk}(\mathcal{M})=\operatorname{Sk}(\mathcal{N})=S$. We define the model $\mathcal{M} \uplus \mathcal{N}=$ $\left\langle M_{w}^{\uplus} \mid w \in W^{\uplus}\right\rangle$, the disjoint union of $\mathcal{M}$ and $\mathcal{N}$, by the following clauses:

- $W^{\uplus}=\operatorname{wrd}(\mathcal{M}) \uplus \operatorname{wrd}(\mathcal{N}) ;^{3}$

- $\operatorname{dom}(\mathcal{M} \uplus \mathcal{N})=\operatorname{dom}(S)$;

- For a function symbol $f, f^{\mathcal{M} \uplus \mathcal{N}}=f^{S}$;

- For a relation symbol $R$ and a world $w \in \operatorname{wrd}(\mathcal{M}), R^{M_{\langle w, 0\rangle}^{\uplus}}=R^{M_{w}}$, and similarly for $\mathcal{N}$;

- For $d_{1}, d_{2} \in \operatorname{dom}(S)$ and a world $w \in \operatorname{wrd}(\mathcal{M}), d_{1} \sim^{M_{\langle w, 0\rangle}^{\uplus}} d_{2}$ if and only if $d_{1} \sim^{M_{w}} d_{2}$, and similarly for $w \in \operatorname{wrd}(\mathcal{N})$.

As a notational convention we will write $w^{\mathcal{M}}$ instead of $\langle w, 0\rangle$. We assume the same notation for information states: for $s \subseteq \operatorname{wrd}(\mathcal{M})$ we will write $s^{\mathcal{M}}$. This notation is particularly useful when we consider the disjoint union of several models with the same set of worlds.

TheOREM 3.12. Given an assignment $g: \operatorname{Var} \rightarrow \operatorname{dom}(S)$ and an info state $s \subseteq \operatorname{wrd}(\mathcal{M})$ of $\mathcal{M}$, then for every formula $\varphi$

$$
\mathcal{M} \uplus \mathcal{N}, s^{\mathcal{M}} \vDash_{g} \varphi \Longleftrightarrow \mathcal{M}, s \vDash_{g} \varphi
$$

Proof. By definition $(\mathcal{M} \uplus \mathcal{N})_{s} \mathcal{M}$ and $\mathcal{M}_{s}$ are the same model-modulo identifying the info states $s^{\mathcal{M}}$ and $s$. The result follows trivially.

The next construction presents a generalization of the disjoint union to models with different skeletons. The new structure obtained will encode the information carried by both models, in a way that lets us recover it naturally.

\footnotetext{
${ }^{3} \operatorname{Recall}$ that $\operatorname{wrd}(\mathcal{M}) \uplus \operatorname{wrd}(\mathcal{N})=(\operatorname{wrd}(\mathcal{M}) \times\{0\}) \times(\operatorname{wrd}(\mathcal{N}) \times\{1\})$.
} 


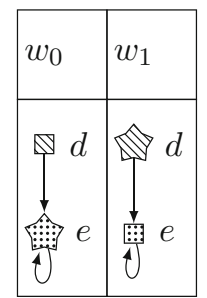

$\oplus$

\begin{tabular}{|c|c|}
\hline$v_{0}$ & $\underset{b}{\mathbb{B}}$ \\
\hline$v_{1}$ & $\underset{a}{\mathbb{N}} \quad \frac{\mathbb{v}}{b}$ \\
\hline
\end{tabular}

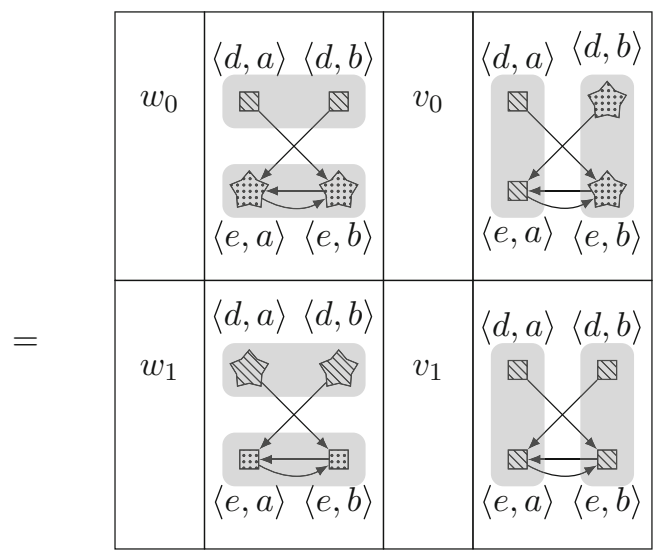

Figure 8. An example of direct sum: the model $\mathcal{M} \oplus \mathcal{N}$ on the right is obtained as the direct sum of the two models on the left, $\mathcal{M}$ (above) and $\mathcal{N}$ (below). For $w \in \operatorname{wrd}(\mathcal{M})$ the projection map on the first component $\pi_{1}: \operatorname{dom}(\mathcal{M}) \times \operatorname{dom}(\mathcal{N}) \rightarrow \operatorname{dom}(\mathcal{M})$ respects the relation $\sim^{\langle w, 1\rangle}$ (i.e., the elements in the same gray box have the same image) and commutes with the interpretation of function symbols. The same holds for $w \in \operatorname{wrd}(\mathcal{N})$ and the projection map on the second component $\pi_{2}$

Definition 3.13. (Direct sum) Let $\mathcal{M}=\left\langle M_{w} \mid w \in W\right\rangle$ and $\mathcal{N}$ be two models. We define the model $\mathcal{M} \oplus \mathcal{N}=\left\langle M_{w}^{\oplus} \mid w \in W^{\oplus}\right\rangle$, the direct sum of $\mathcal{M}$ and $\mathcal{N}$, by the following clauses

- $W^{\oplus}=\operatorname{wrd}(\mathcal{M}) \uplus \operatorname{wrd}(\mathcal{N})$;

- $\operatorname{dom}(\mathcal{M} \oplus \mathcal{N})=\operatorname{dom}(\mathcal{M}) \times \operatorname{dom}(\mathcal{N})$;

- For a function symbol $f, f^{\mathcal{M} \oplus \mathcal{N}}=\left\langle f^{\mathcal{M}}, f^{\mathcal{N}}\right\rangle$;

- For a relation symbol $R$ and a world $w \in \operatorname{wrd}(\mathcal{M})$

$$
R^{M_{\langle w, 1\rangle}^{\oplus}}\left(\left\langle d_{1}, d_{1}^{\prime}\right\rangle, \ldots,\left\langle d_{n}, d_{n}^{\prime}\right\rangle\right) \Longleftrightarrow R^{M_{w}}\left(d_{1}, \ldots, d_{n}\right)
$$

and similarly for $\mathcal{N}$;

- For $d, d^{\prime} \in \operatorname{dom}(S)$ and a world $w \in \operatorname{wrd}(\mathcal{M})$

$$
\left\langle d, d^{\prime}\right\rangle \sim^{M_{\langle w, 1\rangle}^{\oplus}}\left\langle e, e^{\prime}\right\rangle \Longleftrightarrow d \sim^{M_{w}} e
$$

and similarly for $\mathcal{N}$;

We assume the same notational convention for worlds and info states as for the disjoint union. In particular, $w^{\mathcal{M}}$ and $s^{\mathcal{M}}$ refer to a world and an info state of $\mathcal{M}$ respectively. It's worth pointing out that disjoint union preserves 
skeletons while the direct sum does not - see for example the graphical representation of a direct sum model given in Figure 8.

The projections from the domain of this new model to the domain of its components $\left(\pi_{1}: \operatorname{dom}(\mathcal{M}) \times \operatorname{dom}(\mathcal{N}) \rightarrow \operatorname{dom}(\mathcal{M})\right.$ and $\pi_{2}: \operatorname{dom}(\mathcal{M}) \times$ $\operatorname{dom}(\mathcal{N}) \rightarrow \operatorname{dom}(\mathcal{N}))$ commute with the action of assignments and interpretation of terms:

$$
\left(\pi_{1} g\right)(x)=\pi_{1}(g(x)) \quad \pi_{1}\left(t^{\mathcal{M} \oplus \mathcal{N}}\left(a_{1}, \ldots, a_{n}\right)\right)=t^{\mathcal{M}}\left(\pi_{1}\left(a_{1}\right), \ldots, \pi_{1}\left(a_{n}\right)\right)
$$

Given this, by reasoning as in the disjoint union case we obtain a strong connection between $\mathcal{M} \oplus \mathcal{N}$ and the models $\mathcal{M}$ and $\mathcal{N}$.

ThEOREM 3.14. Let $g: \operatorname{Var} \rightarrow \operatorname{dom}(\mathcal{M}) \times \operatorname{dom}(\mathcal{N})$ be an assignment and let $s \subseteq \operatorname{wrd}(\mathcal{M})$ be an info state of $\mathcal{M}$. Then for every formula $\varphi$

$$
\mathcal{M} \oplus \mathcal{N}, s^{\mathcal{M}} \vDash_{g} \varphi \Longleftrightarrow \mathcal{M}, s \vDash_{\pi_{1} g} \varphi
$$

Proof. By definition $(\mathcal{M} \oplus \mathcal{N})_{s} \mathcal{M}=\mathcal{M}_{s} \rtimes \operatorname{Sk}(\mathcal{N})$. The result then follows by Lemma 3.8.

COROLlary 3.15. Using the notations of Theorem 3.14, for $u \subseteq \operatorname{wrd}(\mathcal{M} \oplus$ $\mathcal{N})$

$$
\mathcal{M} \oplus \mathcal{N}, u \vDash_{g} \varphi \Longrightarrow \mathcal{M}, u \cap \operatorname{wrd}(\mathcal{M}) \vDash_{\pi_{1} g} \varphi
$$

ProOF. By persistency we obtain

$$
\begin{aligned}
\mathcal{M} \oplus \mathcal{N}, u \vDash_{g} \varphi & \Longrightarrow \mathcal{M} \oplus \mathcal{N}, u \cap \operatorname{wrd}(\mathcal{M}) \vDash_{g} \varphi \\
& \Longrightarrow \mathcal{M}, u \cap \operatorname{wrd}(\mathcal{M}) \vDash_{\pi_{1} g} \varphi
\end{aligned}
$$

It is straight-forward to generalize Definitions 3.11, 3.13 and Theorems 3.12, 3.14 to arbitrary families of models. We leave the details to the reader and limit ourselves to fix the notations for these constructions.

Let $\left\langle\mathcal{M}_{i} \mid i \in I\right\rangle$ be a family of models. Then:

- If $\forall i, j \in I . \operatorname{Sk}\left(\mathcal{M}_{i}\right)=\operatorname{Sk}\left(\mathcal{M}_{j}\right)$, we indicate with $\biguplus_{i \in I} \mathcal{M}_{i}$ the disjoint union of the family.

- We indicate with $\bigoplus_{i \in I} \mathcal{M}_{i}$ the direct sum of the family.

Studying these constructions we can infer the following preservation results, that will be useful in later Sections of the paper: if we perform one of the above constructions starting from a family of models of a classical formula $\alpha$, by truth-conditionality we obtain again a model of $\alpha$. 
Corollary 3.16 .

$$
\begin{aligned}
& \biguplus_{i \in I} \mathcal{M}^{i} \vDash_{g} \alpha \Longleftrightarrow \forall j \in I . \mathcal{M}^{j} \vDash_{\pi_{j}} \alpha \\
& \bigoplus_{i \in I} \mathcal{M}^{i} \vDash_{g} \alpha \Longleftrightarrow \forall j \in I . \mathcal{M}^{j} \vDash_{\pi_{j} g} \alpha
\end{aligned}
$$

Proof. We only show the second equivalence, as the proof for the first one is completely analogous.

$$
\begin{aligned}
\bigoplus_{i \in I} \mathcal{M}^{i} \vDash_{g} \alpha & \Longleftrightarrow \forall w^{j} \in \biguplus_{j \in I} \operatorname{wrd}\left(\mathcal{M}^{j}\right) \cdot \bigoplus_{i \in I} \mathcal{M}^{i},\left\{w^{j}\right\} \vDash_{g} \alpha \\
& \Longleftrightarrow \forall w^{j} \in \biguplus_{j \in I} \operatorname{wrd}\left(\mathcal{M}^{j}\right) \cdot \mathcal{M}^{j},\left\{w^{j}\right\} \vDash_{\pi_{j} g} \alpha \\
& \Longleftrightarrow \forall j \in I \cdot \mathcal{M}^{j} \vDash_{\pi_{j} g} \alpha
\end{aligned}
$$

\subsection{Characteristic Model}

The next construction we consider allows us to encode all the information entailed by a classical theory, characterizing the set of its theorems. The key to this result is Corollary 3.16.

Recall that a theory is called classical if it contains only classical formulas, that is usual FO formulas. Consider a classical theory $\Gamma$ and define $\mathrm{C}(\Gamma)=$ $\{\varphi \mid \Gamma \not \forall \varphi\}$ as the set of its non-theorems. By definition, for every $\varphi \in \mathrm{C}(\Gamma)$ we can find a pair $\left\langle\mathcal{M}_{\varphi}, g_{\varphi}\right\rangle$ that acts as a witness of the non-entailment $\Gamma \not \forall \varphi$, meaning that $\mathcal{M}_{\varphi} \vDash_{g_{\varphi}} \Gamma$ and $\mathcal{M}_{\varphi} \forall_{g_{\varphi}} \varphi$. Fixing now a family $\left\{\left\langle\mathcal{M}_{\varphi}, g_{\varphi}\right\rangle \mid \varphi \in \mathrm{C}(\Gamma)\right\}$ of models as described, we are ready to define our next construction.

Definition 3.17. (Characteristic model of $\Gamma$ ) Define $\mathcal{M}_{\Gamma}=\bigoplus_{\varphi \in \mathrm{C}(\Gamma)} \mathcal{M}_{\varphi}$ and $g_{\Gamma}: \operatorname{Var} \rightarrow \operatorname{dom}\left(\mathcal{M}_{\Gamma}\right)$ as $g_{\Gamma}(x)=\left\langle g_{\varphi}(x) \mid \varphi \in \mathrm{C}(\Gamma)\right\rangle$.

$\mathrm{C}(\Gamma)$ is a set and not a proper class - since it is a collection of formulas in a fixed signature - and consequently the same holds for $\left\{\left\langle\mathcal{M}_{\varphi}, g_{\varphi}\right\rangle \mid \varphi \in \mathrm{C}(\Gamma)\right\}$.

Moreover, the definition of the characteristic model of $\Gamma$ strongly depends on the set $\left\{\left\langle\mathcal{M}_{\varphi}, g_{\varphi}\right\rangle \mid \varphi \in \mathrm{C}(\Gamma)\right\}$. For our purposes we do not need to exploit this dependence, but its worth noticing that the construction presented here makes use of the axiom of choice.

Theorem 3.18. For every formula $\psi$

$$
\Gamma \vDash \psi \Longleftrightarrow \mathcal{M}_{\Gamma} \vDash_{g_{\Gamma}} \psi
$$


Proof. For the left-to-right implication: From Corollary 3.16 and the definition of $\mathcal{M}_{\Gamma}$ it follows that $\mathcal{M}_{\Gamma} \vDash_{g_{\Gamma}} \Gamma$. By hypothesis $\Gamma \vDash \psi$, and so $\mathcal{M}_{\Gamma} \vDash_{g_{\Gamma}} \psi$, as wanted.

For the right-to-left implication: fix $\varphi$ a non-theorem of $\Gamma$. We claim that the characteristic model does not support $\varphi$. By contradiction, if the model supports the formula then from the generalization of Theorem 3.14 and the persistency of the logic we would obtain

$$
\begin{aligned}
\mathcal{M}_{\Gamma} \vDash_{g_{\Gamma}} \varphi & \Longrightarrow \mathcal{M}_{\Gamma}, \operatorname{wrd}\left(\mathcal{M}_{\varphi}\right) \vDash_{g_{\Gamma}} \varphi \quad\left(\operatorname{as}\left(\mathcal{M}_{\Gamma}\right)_{\operatorname{wrd}\left(\mathcal{M}_{\varphi}\right)}=\mathcal{M}_{\varphi}\right) \\
& \Longrightarrow \mathcal{M}_{\varphi} \vDash_{g_{\varphi}} \varphi
\end{aligned}
$$

which gives a contradiction, as wanted.

This result gives us a non-trivial property of InqBQ with respect to FO: given an arbitrary classical theory $\Gamma$, we can find a single model which entail all and only the theorems of $\Gamma$. It is well-known that the same property does not hold for the standard semantics of FO, as the set of formulas supported by a classical structure is necessarily a complete theory. We will see that this property is of fundamental importance for the proof of the generalized existence property presented in Section 4.

The same result does not hold for a generic theory. Consider for example

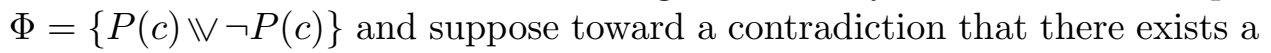
pair $\langle\mathcal{M}, g\rangle$ such that

$$
\Phi \vDash \psi \Longleftrightarrow \mathcal{M} \vDash_{g} \psi
$$

Then it is clear that $\mathcal{M} \vDash_{g} P(c)$ or $\mathcal{M} \vDash_{g} \neg P(c)$ by the semantic clause for $\mathbb{V}$. But $\Phi \not \forall P(c)$ and $\Phi \not \forall \neg P(c)$, thus we have a contradiction.

\subsection{Permutation Models}

We introduce now some constructions based on permutations. These constructions will be the key ingredient to prove the existence property in the following section.

A simple way to obtain a new model is by simply swapping the names of the elements. This is the basic idea behind the following construction.

With $\mathfrak{p}(X)$ we will indicate the set of permutations of $X$, that is the bijective functions $\sigma: X \rightarrow X$.

Definition 3.19. Let $S$ be a skeleton and $\sigma \in \mathfrak{p}(\operatorname{dom}(S))$ a permutation. We define the skeleton $S^{\sigma}$ by the following clauses

- $\operatorname{dom}\left(S^{\sigma}\right)=\operatorname{dom}(S)$;

- $f^{S^{\sigma}}\left(d_{1}, \ldots, d_{n}\right)=\sigma\left(f^{S}\left(\sigma^{-1} d_{1}, \ldots, \sigma^{-1} d_{n}\right)\right)$. 

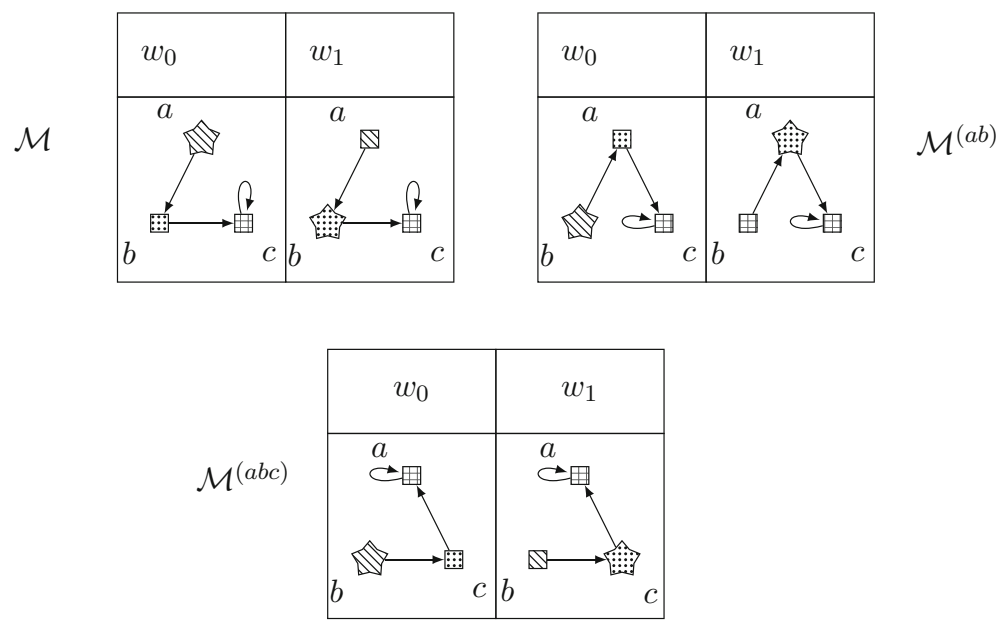

Figure 9. Examples of permutation models. With the notation $\left(a_{1} a_{2} \ldots a_{n}\right)$ we indicate the $n$-cycle mapping $a_{1}$ to $a_{2}, a_{2}$ to $a_{3}$ and so on. In the Figure we depict the models $\mathcal{M}$ (upper left), $\mathcal{M}^{(a b)}$ (upper right) and $\mathcal{M}^{(a b c)}$. The roles of the elements in the models are swapped according to the inverse of the permutation considered. For example, the element $a$ in $\mathcal{M}^{(a b c)}$ behaves like the element $c$ in $\mathcal{M}$, as $a$ is mapped onto $c$ by the permutation $(a b c)^{-1}$

Definition 3.20. (Permutation model) Given a model $\mathcal{M}=\left\langle M_{w} \mid w \in W\right\rangle$ we define the model $\mathcal{M}^{\sigma}=\left\langle M_{w}^{\sigma} \mid w \in W^{\sigma}\right\rangle$ by the following clauses

- $W^{\sigma}=W$

- $\operatorname{Sk}\left(\mathcal{M}^{\sigma}\right)=\operatorname{Sk}(\mathcal{M})^{\sigma}$

- $R^{M_{w}^{\sigma}}\left(d_{1}, \ldots, d_{n}\right)$ iff $R^{M_{w}}\left(\sigma^{-1} d_{1}, \ldots, \sigma^{-1} d_{n}\right)$;

- $d_{1} \sim^{M_{w}^{\sigma}} d_{2}$ iff $\sigma^{-1} d_{1} \sim^{M_{w}} \sigma^{-1} d_{2}$.

In Figure 9 two examples of permutation models are depicted.

TheOREM 3.21. Let $s \subseteq \operatorname{wrd}(\mathcal{M})$ be an info state and $g: \operatorname{Var} \rightarrow \operatorname{dom}(\mathcal{M})$ an assignment. Then for every formula $\varphi$

$$
\mathcal{M}, s \vDash_{g} \varphi \Longleftrightarrow \mathcal{M}^{\sigma}, s \vDash_{\sigma g} \varphi
$$

Proof. The functions id : $\operatorname{wrd}(\mathcal{M}) \rightarrow \operatorname{wrd}\left(\mathcal{M}^{\sigma}\right)$ and $\sigma: \operatorname{dom}(\mathcal{M}) \rightarrow$ $\operatorname{dom}\left(\mathcal{M}^{\sigma}\right)$ respect the hypothesis of Lemma 3.4, thus the result follows.

As we saw with the previous construction, we can consider a permutation $\sigma$ over the domain of a model $\mathcal{M}$, and this gives us a way to define a new model $\mathcal{M}^{\sigma}$. It is not hard to define from here an action of the group $\mathfrak{p}(\operatorname{dom}(\mathcal{M}))$ 
over the set $\left\{\mathcal{M}^{\sigma} \mid \sigma \in \mathfrak{p}(\operatorname{dom}(\mathcal{M}))\right\}$. What is surprising, is that this action can be encoded by a single information model obtained "gluing together" the term models of each $\mathcal{M}^{\sigma}$. This construction allows us to study which properties expressed by InqBQ are preserved under the action presented above and gives us the tools needed to prove the existence property.

Observe that a permutation $\sigma \in \mathfrak{p}(\operatorname{dom}(\mathcal{M}))$ can be extended to $\tilde{\sigma} \in$ $\mathfrak{p}(\Sigma[\operatorname{dom}(\mathcal{M})])$ by the following clauses:

- For $d \in \operatorname{dom}(\mathcal{M}), \tilde{\sigma}(\underline{d})=\underline{\sigma(d)}$;

- For $f$ a function symbol, $\tilde{\sigma}\left(f\left(t_{1}, \ldots, t_{n}\right)\right)=f\left(\tilde{\sigma}\left(t_{1}\right), \ldots, \tilde{\sigma}\left(t_{n}\right)\right)$.

This observation leads naturally to the following definitions:

DEFinition 3.22. (Full permutation model)

- Given $\sigma \in \mathfrak{p}(\operatorname{dom}(\mathcal{M}))$, we define the model $\mathbf{T}^{\sigma} \mathcal{M}=(\mathbf{T} \mathcal{M})^{\tilde{\sigma}}$.

- Let $\mathcal{M}$ be a model. We define its full permutation model as

$$
\mathfrak{p} \mathcal{M}=\biguplus_{\sigma \in \mathfrak{p}(\operatorname{dom}(\mathcal{M}))} \mathbf{T}^{\sigma} \mathcal{M}
$$

A graphical representation of a model $\mathbf{T}^{\sigma} \mathcal{M}$ is given in Figure 10. To simplify the notation, we will write $w^{\sigma}$ and $s^{\sigma}$ instead of $w^{\mathbf{T}^{\sigma} \mathcal{M}}$ and $s^{\mathbf{T}^{\sigma} \mathcal{M}}$ to refer to words and info states in $\mathbf{T}^{\sigma} \mathcal{M}$.

For $\mathfrak{p} \mathcal{M}$ to be well-defined, the condition $\operatorname{Sk}\left(\mathbf{T}^{\sigma} \mathcal{M}\right)=\operatorname{Sk}(\mathbf{T} \mathcal{M})$ needs to hold. And this is in fact the case, as:

$$
\begin{aligned}
f^{\mathbf{T}^{\sigma} \mathcal{M}}\left(t_{1}, \ldots, t_{n}\right) & =\tilde{\sigma} f^{\mathbf{T} \mathcal{M}}\left(\tilde{\sigma}^{-1} t_{1}, \ldots, \tilde{\sigma}^{-1} t_{n}\right) \\
& =\tilde{\sigma} f\left(\tilde{\sigma}^{-1} t_{1}, \ldots, \tilde{\sigma}^{-1} t_{n}\right) \\
& =f\left(\tilde{\sigma} \tilde{\sigma}^{-1} t_{1}, \ldots, \tilde{\sigma} \tilde{\sigma}^{-1} t_{n}\right) \\
& =f\left(t_{1}, \ldots, t_{n}\right) \\
& =f^{\mathbf{T} \mathcal{M}}\left(t_{1}, \ldots, t_{n}\right)
\end{aligned}
$$

Corollary 3.23. Let $\mathcal{M}=\left\langle M_{w} \mid w \in W\right\rangle$ be a model with domain $D, s \subseteq$ $W$ an info state and $g: \operatorname{Var} \rightarrow \Sigma[D]$ an assignment.

1. For every formula $\varphi$ it holds

$$
\mathbf{T}^{\sigma} \mathcal{M}, s \vDash_{\tilde{\sigma} g} \varphi \Longleftrightarrow \mathcal{M}, s \vDash_{\tau \circ g} \varphi
$$

2. Let $\sigma \in \mathfrak{p}(D)$ a permutation. Then for every formula $\varphi$

$$
\mathfrak{p} \mathcal{M}, s^{\sigma} \vDash_{\tilde{\sigma} g} \varphi \Longleftrightarrow \mathcal{M}, s \vDash_{\tau \circ g} \varphi
$$



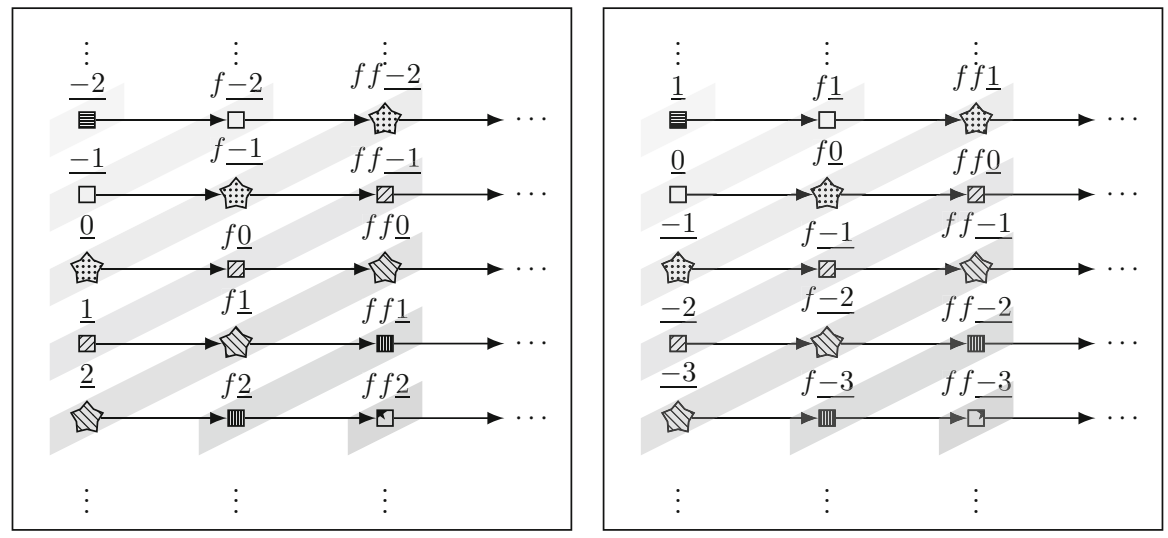

Figure 10. An example of a permutation model. On the left, the model $\mathbf{T} \mathcal{M}$ of Figure 6 . On the right, its corresponding permutation model $\mathbf{T}^{\sigma} \mathcal{M}$ for $\sigma(n)=-n-1$. The skeleton is preserved by this operation, while the interpretation of predicate $P$ and of identity changes in accordance with the permutation. For example, $\underline{0} \sim^{\mathbf{T M}} f \underline{-1}$, but $\underline{0} \varkappa^{\mathbf{T}^{\sigma} \mathcal{M}}$ $f-1$

Proof. The first statement follows combining Theorems 3.10 and 3.21.

The second statement follows trivially from the first one, as $(\mathfrak{p} \mathcal{M})_{s^{\sigma}}=$ $\left(\mathbf{T}^{\sigma} \mathcal{M}\right)_{s}$.

COROllary 3.24. In the same notations as Corollary 3.23; consider $\rho \in$ $\mathfrak{p}(D)$ a permutation. Define $\rho s=\left\{w^{\rho \sigma} \mid w^{\sigma} \in s\right\}{ }^{4}$ Then for every formula $\varphi$

$$
\mathfrak{p} \mathcal{M}, s \vDash_{G} \varphi \Longleftrightarrow \mathfrak{p} \mathcal{M}, \rho s \vDash_{\rho G} \varphi
$$

Proof. Define $F_{\rho}: W \rightarrow W$ as $F_{\rho}\left(w^{\sigma}\right):=w^{\rho \sigma} . F_{\rho}$ and $\tilde{\rho}: \Sigma[D] \rightarrow \Sigma[D]$ respect the hypothesis of Lemma 3.4, thus the thesis follows.

In Figure 11 we give a graphical summary of the constructions introduced.

\section{Disjunction and Existence Properties}

\subsection{Disjunction Property}

We make use of the constructions introduced to prove that the logic InqBQ satisfies a strong form of the disjunction property, namely the disjunction property over classical theories.

\footnotetext{
${ }^{4}$ Here the notation $\rho \sigma$ indicates the composition of the permutations $\rho$ and $\sigma$.
} 


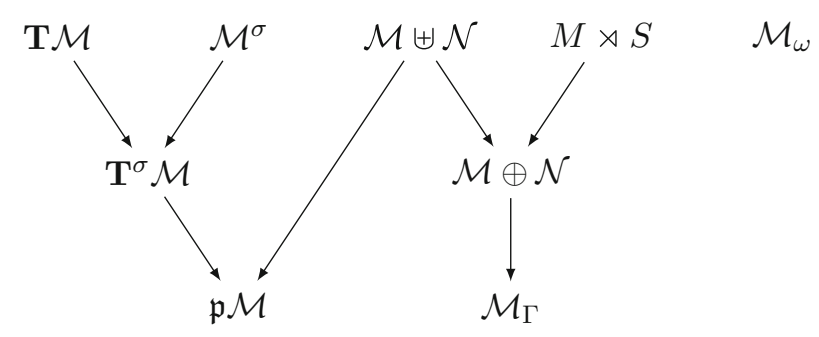

Figure 11. Summary of the constructions introduced. An arrow connects two constructions if the source was used to define the target

TheOREm 4.1. Given a classical theory $\Gamma$ (i.e., $\Gamma \subseteq$ FO), for every formulas $\varphi$ and $\psi$

$$
\Gamma \vDash \varphi \llbracket \psi \Longrightarrow \Gamma \vDash \varphi \text { or } \Gamma \vDash \psi
$$

Proof. Suppose $\Gamma \not \models \varphi$ and $\Gamma \not \forall \psi$. Let $\mathcal{M}_{\varphi}, \mathcal{M}_{\psi}$ be two models and $g_{\varphi}, g_{\psi}$ be two assignments such that

$$
\left\{\begin{array} { l } 
{ \mathcal { M } _ { \varphi } \vDash _ { g _ { \varphi } } \Gamma } \\
{ \mathcal { M } _ { \varphi } \nvdash _ { g _ { \varphi } } \varphi }
\end{array} \quad \left\{\begin{array}{l}
\mathcal{M}_{\psi} \vDash_{g_{\psi}} \Gamma \\
\mathcal{M}_{\psi} \forall_{g_{\psi}} \psi
\end{array}\right.\right.
$$

Then consider the model $\mathcal{M}_{\varphi} \oplus \mathcal{M}_{\psi}$ and the assignment $G=\left\langle g_{\varphi}, g_{\psi}\right\rangle$. By Corollary 3.16 it follows that $\mathcal{M}_{\varphi} \oplus \mathcal{M}_{\psi} \vDash_{G} \Gamma$. Moreover by Theorem 3.14 and persistency it follows

$$
\begin{aligned}
\mathcal{M}_{\varphi} \not_{g_{\varphi}} \varphi & \Longrightarrow \mathcal{M}_{\varphi} \oplus \mathcal{M}_{\psi}, \operatorname{wrd}\left(\mathcal{M}_{\varphi}\right) \not_{G} \varphi \\
& \Longrightarrow \mathcal{M}_{\varphi} \oplus \mathcal{M}_{\psi} \forall_{G} \varphi
\end{aligned}
$$

and with an similar argument we obtain $\mathcal{M}_{\varphi} \oplus \mathcal{M}_{\psi} \forall_{G} \psi$ too. From this it follows $\Gamma \not \varphi \vee \vee \psi$.

Another possible proof of the theorem could be carried out considering the characteristic model of $\Gamma$ instead of the models $\mathcal{M}_{\varphi}$ and $\mathcal{M}_{\psi}$. The advantage of the proof presented above is that it does not make use of the axiom of choice.

\subsection{Existence Property}

In a similar fashion as above, even though the proof is a bit more involved, we can prove that InqBQ satisfies a strong form of the existence property, namely the existence property for classical theories.

The proof will make extensive use of the model $\mathfrak{p}\left(\left(\mathcal{M}_{\Gamma}\right)_{\omega}\right)$, so it is useful to explicitly state what are the components of this model. Define $\mathcal{P}=$ $\mathfrak{p}\left(\left(\mathcal{M}_{\Gamma}\right)_{\omega}\right)=\left\langle P_{w}^{\sigma} \mid\langle w, \sigma\rangle \in W^{P}\right\rangle$. Then: 
- $W^{P}=\biguplus_{\sigma \in \mathfrak{p}\left(\operatorname{dom}\left(\mathcal{M}_{\Gamma}\right) \times \omega\right)} \operatorname{wrd}\left(\mathcal{M}_{\Gamma}\right)$.

- $\operatorname{dom}(\mathcal{P})=\Sigma\left[\operatorname{dom}\left(\mathcal{M}_{\Gamma}\right) \times \omega\right]$.

- $f^{\mathcal{P}}\left(t_{1}, \ldots, t_{n}\right)=f\left(t_{1}, \ldots, t_{n}\right)$.

- $R^{P_{w}^{\sigma}}\left(t_{1}, \ldots, t_{n}\right)$ if and only if $R^{\left(\left(\mathcal{M}_{\Gamma}\right)_{\omega}\right)_{w}}\left(\sigma^{-1}\left(t_{1}\right), \ldots, \sigma^{-1}\left(t_{n}\right)\right)$ if and only if $R^{\left(\mathcal{M}_{\Gamma}\right)_{w}}\left(\pi_{1} \sigma^{-1}\left(t_{1}\right), \ldots, \pi_{1} \sigma^{-1}\left(t_{n}\right)\right)$.

Notice that the elements $\langle d, k\rangle,\left\langle d, k^{\prime}\right\rangle \in \operatorname{dom}\left(\mathcal{M}_{\Gamma}\right) \times \omega$ play the same role in the model $\left(\mathcal{M}_{\Gamma}\right)_{\omega}$, but they can still be mapped on elements supporting different formulas by a permutation in $\mathfrak{p}\left(\operatorname{dom}\left(\mathcal{M}_{\Gamma}\right) \times \omega\right)$. This will be a key element of the proof.

THEOREM 4.2. Given a classical theory $\Gamma$, then for every formula $\bar{\exists} x . \varphi(x)$

$$
\Gamma \vDash \bar{\exists} x . \varphi(x) \Longrightarrow \Gamma \vDash \varphi(t) \text { for some term } t
$$

Proof. Without loss of generality we can suppose $\Gamma$ to be closed under logical entailment. ${ }^{5}$

Fix $\varphi\left(x, y_{1}, \ldots, y_{m}\right)$ (where $x, y_{1}, \ldots, y_{m}$ is a complete list of the distinct free variables in $\varphi$ ) and suppose that $\Gamma \not \forall \varphi\left(t, y_{1}, \ldots, y_{m}\right)$ for every term $t$. Consider the characteristic model $\mathcal{M}_{\Gamma}$ of the theory $\Gamma$ and the assignment $g_{\Gamma}: \operatorname{Var} \rightarrow \operatorname{dom}\left(\mathcal{M}_{\Gamma}\right)$ such that $\Gamma \vDash \psi$ iff $\mathcal{M}_{\Gamma} \vDash_{g_{\Gamma}} \psi$. Our aim is now, manipulating the model $\mathcal{M}_{\Gamma}$, to build a model of $\Gamma$ but not of the formula $\bar{\exists} x . \varphi(x, \bar{y})$, thus proving that $\Gamma \not \bar{\exists} x \cdot \varphi(x, \bar{y})$.

As such a model consider $\mathcal{P}$. This is a model of $\Gamma$ as we have

$$
\begin{array}{rlr} 
& \mathcal{M}_{\Gamma} \vDash \Gamma & \\
\Longleftrightarrow & \forall w \in \operatorname{wrd}\left(\mathcal{M}_{\Gamma}\right) \cdot \mathcal{M}_{\Gamma},\{w\} \vDash \Gamma & \text { by Lemma } 2.11 \\
\Longleftrightarrow & \forall w \in \operatorname{wrd}\left(\left(\mathcal{M}_{\Gamma}\right)_{\omega}\right) \cdot\left(\mathcal{M}_{\Gamma}\right)_{\omega},\{w\} \vDash \Gamma & \text { by Lemma } 3.6 \\
\Longleftrightarrow & \forall \sigma \in \mathfrak{p}\left(\operatorname{dom}\left(\left(\mathcal{M}_{\Gamma}\right)_{\omega}\right)\right) \cdot \forall w^{\sigma} \in \operatorname{wrd}\left(\left(\mathcal{M}_{\Gamma}\right)_{\omega}\right) \cdot \mathcal{P},\left\{w^{\sigma}\right\} \vDash \Gamma \\
\Longleftrightarrow & \text { by Corollary } 3.23 \\
\Longleftrightarrow & \forall v \in \operatorname{wrd}(\mathcal{P}) \cdot \mathcal{P},\{v\} \vDash \Gamma & \\
& & \text { by Lemma } 2.11
\end{array}
$$

\footnotetext{
${ }^{5}$ It is trivial to prove that for InqBQ, as for FO, entailment is invariant under substitutions of variables with fresh constants. Formally, consider a set of formulas $\Phi \cup\{\psi\}$ and a partial variable substitution $f: \operatorname{Var} \rightarrow C$ for $C$ a set of constants not appearing in $\Phi \cup\{\psi\}$. Define $\Phi[f]$ and $\psi[f]$ as the result of applying the substitution to the set $\Phi$ and $\psi$ in the usual way. Then it holds $\Phi \vDash \psi$ iff $\Phi[f] \vDash \psi[f]$.
} 
Towards a contradiction suppose that $\Gamma \vDash \bar{\exists} x \cdot \varphi(x, \bar{y})$. So for a certain element $t\left(\underline{\left\langle d_{1}, k_{1}\right\rangle}, \ldots,\left\langle\underline{\left\langle d_{n}, k_{n}\right\rangle}\right) \in \operatorname{dom}(\mathcal{P})=\Sigma\left[\operatorname{dom}\left(\left(\mathcal{M}_{\Gamma}\right)_{\omega}\right)\right]\right.$ (where we suppose the elements $\left\langle d_{i}, k_{i}\right\rangle$ to be distinct) we have

$$
\mathcal{P} \vDash_{h} \varphi\left(x, y_{1}, \ldots, y_{m}\right) \quad \text { if } \quad h(x)=t\left(\underline{\left\langle d_{1}, k_{1}\right\rangle}, \ldots, \underline{\left\langle d_{n}, k_{n}\right\rangle}\right)
$$

from which it follows that

$$
\mathcal{P} \vDash_{h} \varphi\left(t\left(z_{1}, \ldots, z_{n}\right), y_{1}, \ldots, y_{m}\right) \quad \text { if } \quad h\left(z_{i}\right)=\underline{\left\langle d_{i}, k_{i}\right\rangle}
$$

where $z_{1}, \ldots, z_{n}$ are fresh variables distinct from $y_{1}, \ldots, y_{m}$.

Fix now the assignment $H$ such that $H\left(z_{i}\right)=\left\langle g_{\Gamma}\left(z_{i}\right), i\right\rangle$ for $1 \leq i \leq n$ and $H\left(y_{j}\right)=\left\langle g_{\Gamma}\left(y_{j}\right), n+j\right\rangle$ for $1 \leq j \leq m$. As $H$ is injective over the set $\left\{z_{1}, \ldots, z_{n}\right\}$, it is possible to find a permutation $\sigma \in \mathfrak{p}\left(\operatorname{dom}\left(\left(\mathcal{M}_{\Gamma}\right)_{\omega}\right)\right)$ such that $\sigma\left(\underline{\left\langle g_{\Gamma}\left(z_{i}\right), i\right\rangle}\right)=\underline{\left\langle d_{i}, k_{i}\right\rangle}$ for $1 \leq i \leq n .^{6}$

To conclude the proof, consider now the following steps

$$
\begin{array}{rlr} 
& \mathcal{P} \vDash_{\sigma H} \varphi(t(\bar{z}), \bar{y}) & \text { by Equation } 1 \\
\Longrightarrow & \mathcal{P} \vDash_{H} \varphi(t(\bar{z}), \bar{y}) & \text { by Corollary } 3.24 \\
\Longrightarrow & \mathcal{P}, \operatorname{wrd}\left(\left(\mathcal{M}_{\Gamma}\right)_{\omega}\right) \vDash_{H} \varphi(t(\bar{z}), \bar{y}) & \text { by persistency } \\
\Longrightarrow & \left(\mathcal{M}_{\Gamma}\right)_{\omega} \vDash_{\tau \circ H} \varphi(t(\bar{z}), \bar{y}) & \text { by Corollary } 3.23 \\
\Longrightarrow \mathcal{M}_{\Gamma} \vDash_{g_{\Gamma}} \varphi(t(\bar{z}), \bar{y}) & \text { by Lemma } 3.6 \\
\Longrightarrow \Gamma \vDash \varphi(t(\bar{z}), \bar{y}) & \text { by Theorem } 3.18
\end{array}
$$

and this is a contradiction, since it goes against the initial hypothesis that $\Gamma \not \forall \varphi(t, \bar{y})$ for any term $t$. Thus the hypothesis $\Gamma \vDash \bar{\exists} x \cdot \varphi(x, \bar{y})$ does not hold, as wanted.

The following result is a simple corollary of the general form of the existence property, and shows the strongly constructive character of the logic InqBQ.

Theorem 4.3. Let $\varphi\left(x_{1}, \ldots, x_{n}, y\right)$ be a formula and $\Gamma$ a classical theory. Suppose that

$$
\Gamma \vDash \forall \bar{x} \cdot \bar{\exists} y \cdot \varphi(\bar{x}, y)
$$

Then there exists a term $t\left(x_{1}, \ldots, x_{n}\right)$ such that

$$
\Gamma \vDash \forall \bar{x} \cdot \varphi(\bar{x}, t(\bar{x}))
$$

\footnotetext{
${ }^{6}$ This passage justifies the fact that we are considering the model $\mathcal{P}$ instead of the model $\mathfrak{p}\left(\mathcal{M}_{\Gamma}\right)$. Indeed, if $g_{\Gamma}\left(z_{i}\right)=g_{\Gamma}\left(z_{j}\right)$ for $i \neq j$ then it would not be possible to find a permutation $\sigma$ as above. A natural question (currently open) is if $\mathfrak{p}\left(\mathcal{M}_{\Gamma}\right) \not \forall \bar{\exists} x . \varphi(x)$ generally holds.
} 
Proof. In this proof we will adopt a slightly different notation to make explicit reference to the signature adopted: with $\vDash_{\Sigma}$ we indicate the entailment relation of InqBQ relative to the signature $\Sigma$.

Consider $c_{1}, \ldots, c_{n}$ fresh constant symbols not appearing in $\Gamma \cup \varphi$ and $\Sigma_{\bar{c}}$ the signature obtained by adding the new constants to the signature $\Sigma$. Then we have

$$
\begin{aligned}
\Gamma \vDash_{\Sigma} \forall \bar{x} \cdot \bar{\exists} y \cdot \varphi(\bar{x}, y) & \Longleftrightarrow \Gamma \vDash_{\Sigma_{\bar{c}}} \bar{\exists} y \cdot \varphi(\bar{c}, y) \\
& \Longleftrightarrow \Gamma \vDash_{\Sigma_{\bar{c}}} \varphi(\bar{c}, t(\bar{c})) \text { for some } t \\
& \Longleftrightarrow \Gamma \vDash_{\Sigma} \forall \bar{x} \cdot \varphi(\bar{x}, t(\bar{x})) \text { for some } t
\end{aligned}
$$

This theorem has an interesting interpretation connected to the notion of function definability. In the FO case, we say that a formula $\varphi\left(x_{1}, \ldots, x_{n}, y\right)$ defines a function under a classical theory $\Gamma$ if the following entailment holds

$$
\Gamma \vDash \forall \bar{x} \cdot \exists ! y \cdot \varphi(\bar{x}, y)
$$

that is if $\varphi(\bar{x}, y)$ identifies a function in every classical model of $\Gamma$.

In InqBQ we can consider a stronger notion of function definability associated to the inquisitive quantifier. We say that a formula $\varphi(\bar{x}, y)$ strongly defines a function under a classical theory $\Gamma$ if the following entailment holds

$$
\Gamma \vDash \forall \bar{x} . \bar{\exists} ! y \cdot \varphi(\bar{x}, y)
$$

where we have substituted the symbol $\exists$ with $\bar{\exists}$. In particular, the condition implies that in every model $\mathcal{M}$ of the theory $\Gamma, \varphi(\bar{x}, y)$ identifies the same function in every world of $\mathcal{M}$. Theorem 4.3 gives us then a complete characterization of which formulas strongly define a function, that is only the ones that identify the interpretation of a fixed term of the language.

\section{Further Refinements}

It is worth noticing that the proof of the disjunction property given in Theorem 4.1 can be split into two passages:

1. Given two models $\mathcal{M}$ and $\mathcal{N}$ of a classical theory $\Gamma$, then $\mathcal{M} \oplus \mathcal{N}$ is a model of $\Gamma$ too;

2. Given two models $\mathcal{M}_{\varphi}$ and $\mathcal{M}_{\psi}$ of $\Gamma$ such that $\mathcal{M}_{\varphi} \not \forall \varphi$ and $\mathcal{M}_{\psi} \not \forall \psi$, then $\mathcal{M}_{\varphi} \oplus \mathcal{M}_{\psi} \not \forall \varphi \mathbb{\psi} \psi$ by persistency. 


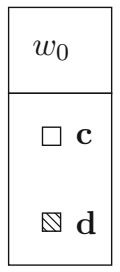

\begin{tabular}{|c|c|}
\hline$\left\langle w_{0}, 0\right\rangle$ & $\left\langle w_{0}, 1\right\rangle$ \\
\hline$\square\langle\mathbf{c}, \mathbf{c}\rangle \mathbb{\mathbb { }}\langle\mathbf{d}, \mathbf{c}\rangle$ & $\square\langle\mathbf{c}, \mathbf{c}\rangle \square\langle\mathbf{d}, \mathbf{c}\rangle$ \\
\hline$\square\langle\mathbf{c}, \mathbf{d}\rangle \mathbb{\mathbb { V }}\langle\mathbf{d}, \mathbf{d}\rangle$ & $\mathbb{\mathbb { N }}\langle\mathbf{c}, \mathbf{d}\rangle \mathbb{\mathbb { N }}\langle\mathbf{d}, \mathbf{d}\rangle$ \\
\hline
\end{tabular}

Figure 12. The models used in the proof of Lemma 5.4

This leads naturally to the following definition.

Definition 5.1. ( $\oplus$ property) Given a theory $\Phi$, we say that it has the $\oplus$ property if

$$
\text { If } \mathcal{M} \vDash_{g} \Phi \text { and } \mathcal{N} \vDash_{h} \Phi \text { then } \mathcal{M} \oplus \mathcal{N} \vDash_{\langle g, h\rangle} \Phi
$$

Given a formula $\varphi$ we say it has the $\oplus$ property if and only if the theory $\{\varphi\}$ has the $\oplus$ property.

COROLlary 5.2. Every classical theory has the $\oplus$ property.

Proof. This is a direct consequence of Corollary 3.16.

And with the same proof as in Theorem 4.1 we obtain the following corollary.

COROLlary 5.3. If a theory $\Phi$ has the $\oplus$ property, then it has the disjunction property.

This also leads to the question if the $\oplus$ property and the disjunction property actually coincide. The answer is no, as we will show with the next results.

Lemma 5.4. There exists a theory with the disjunction property and without the $\oplus$ property.

Proof. Consider the signature $\{c, d\}$ with $c, d$ constant symbols, the model $\mathcal{M}$ depicted in Figure 12 on the left and the set $\operatorname{Th}(\mathcal{M})=\{\varphi \mid \mathcal{M} \vDash \varphi\}$. This theory clearly has the disjunction property as

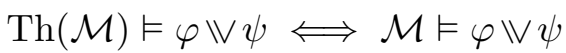

$$
\begin{aligned}
& \Longleftrightarrow \mathcal{M} \vDash \varphi \text { or } \mathcal{M} \vDash \psi \\
& \Longleftrightarrow \operatorname{Th}(\mathcal{M}) \vDash \varphi \text { or } \operatorname{Th}(\mathcal{M}) \vDash \psi
\end{aligned}
$$

Moreover it does not have the $\oplus$ property as the formula

$$
\forall x . \forall y \cdot(x=y \llbracket x \neq y)
$$

is supported at $\mathcal{M}$ but not at $\mathcal{M} \oplus \mathcal{M}$ (Figure 12 on the right). 
Using Corollary 5.3 we can extend Theorem 4.1 to a larger class of theories, namely the $q \mathbb{V}$-free theories.

Definition 5.5. (q $\mathbb{V}$-free formulas) A formula of InqBQ is called quasi $\mathbb{V}$ free ( $\mathrm{q} \vee$-free) if it is generated by the following grammar (where $\alpha$ ranges over classical formulas and $\varphi$ ranges over arbitrary formulas of InqBQ).

$$
\psi::=\alpha|\psi \wedge \psi| \varphi \rightarrow \psi|\bar{\exists} x . \psi| \forall x . \psi
$$

A theory is called $q \mathbb{V}$-free if it contains only $q \mathbb{V}$-free formulas.

Intuition 5.6. Basically a formula is $\mathrm{q} \mathbb{V}$-free if and only if all the occurrences of the symbol $\mathbb{V}$, if there is any, appear in the antecedent of an implication.

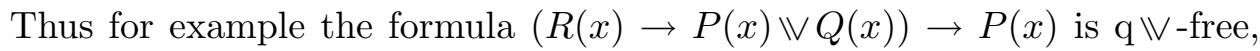
while the formula $P(x) \rightarrow(R(x) \rightarrow P(x) \mathbb{\vee} Q(x))$ is not $\mathrm{q} \mathbb{\vee}$-free.

REMARK 5.7. This fragment is strictly more expressive than the classical fragment. For example the formula $\bar{\exists} x . P(x)$ is not truth-conditional, and so it is not equivalent to any classical formula (compare Lemma 2.11).

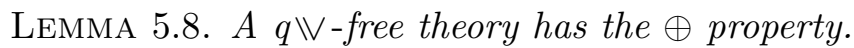

Proof. We will show by induction on the structure of $\varphi$ an $q \mathbb{V}$-free formula that $\varphi$ has the $\oplus$ property. In the rest of the proof $\mathcal{M}$ and $\mathcal{N}$ will indicate two arbitrary information structures; $g$ and $h$ will indicate two arbitrary assignments on $\mathcal{M}$ and $\mathcal{N}$ respectively.

Case $\varphi \equiv \alpha$ this case coincides with Corollary 5.2.

Case $\varphi \equiv \psi \wedge \chi$

$$
\begin{aligned}
& \mathcal{M} \vDash_{g} \psi \wedge \chi \text { and } \mathcal{N} \vDash_{g} \psi \wedge \chi \\
& \Longleftrightarrow \mathcal{M} \vDash_{g} \psi \text { and } \mathcal{M} \vDash_{g} \chi \text { and } \mathcal{N} \vDash_{h} \psi \text { and } \mathcal{N} \vDash_{h} \chi \\
& \quad \text { (by inductive hypothesis) } \\
& \Longleftrightarrow \mathcal{M} \oplus \mathcal{N} \vDash_{\langle g, h\rangle} \psi \text { and } \mathcal{M} \oplus \mathcal{N} \vDash_{\langle g, h\rangle} \chi \\
& \Longleftrightarrow \mathcal{M} \oplus \mathcal{N} \vDash_{\langle g, h\rangle} \psi \wedge \chi
\end{aligned}
$$

Case $\varphi \equiv \psi \rightarrow \chi$

$$
\begin{gathered}
\mathcal{M} \vDash_{g} \psi \rightarrow \chi \text { and } \mathcal{N} \vDash_{h} \psi \rightarrow \chi \\
\Longleftrightarrow \forall s \subseteq \operatorname{wrd}(\mathcal{M}) .\left[\mathcal{M}_{s} \vDash_{g} \psi \Longrightarrow \mathcal{M}_{s} \vDash_{g} \chi\right] \text { and } \\
\forall t \subseteq \operatorname{wrd}(\mathcal{N}) .\left[\mathcal{N}_{t} \vDash_{h} \psi \Longrightarrow \mathcal{N}_{t} \vDash_{h} \chi\right]
\end{gathered}
$$

(by Corollary 3.15 and inductive hypothesis)

$\Longrightarrow \forall s \subseteq \operatorname{wrd}(\mathcal{M}) . \forall t \subseteq \operatorname{wrd}(\mathcal{N})$. 


$$
\begin{gathered}
{\left[\mathcal{M}_{s} \oplus \mathcal{N}_{t} \vDash_{\langle g, h\rangle} \psi \Longrightarrow \mathcal{M}_{s} \oplus \mathcal{N}_{t} \vDash_{\langle g, h\rangle} \chi\right]} \\
\Longleftrightarrow \forall u \subseteq \operatorname{wrd}(\mathcal{M} \oplus \mathcal{N}) .\left[(\mathcal{M} \oplus \mathcal{N})_{u} \vDash_{\langle g, h\rangle} \psi \Longrightarrow(\mathcal{M} \oplus \mathcal{N})_{u} \vDash_{\langle g, h\rangle} \chi\right] \\
\Longleftrightarrow \mathcal{M} \oplus \mathcal{N} \vDash_{\langle g, h\rangle} \psi \rightarrow \chi
\end{gathered}
$$

Case $\varphi \equiv \bar{\exists} x . \psi$

$$
\begin{aligned}
& \mathcal{M} \vDash_{g} \bar{\exists} x \cdot \psi \text { and } \mathcal{N} \vDash_{h} \bar{\exists} x \cdot \psi \\
\Longleftrightarrow & \exists d \in \operatorname{dom}(\mathcal{M}) . \mathcal{M} \vDash_{g[x \mapsto d]} \psi \text { and } \exists e \in \operatorname{dom}(\mathcal{N}) . \mathcal{N} \vDash_{h[x \mapsto e]} \psi \\
\Longleftrightarrow & \exists d \in \operatorname{dom}(\mathcal{M}) . \exists e \in \operatorname{dom}(\mathcal{N}) .\left[\mathcal{M} \vDash_{g[x \mapsto d]} \psi \text { and } \mathcal{N} \vDash_{h[x \mapsto e]} \psi\right]
\end{aligned}
$$

(by inductive hypothesis)

$$
\begin{aligned}
& \Longleftrightarrow \exists\langle d, e\rangle \in \operatorname{dom}(\mathcal{M} \oplus \mathcal{N}) . \mathcal{M} \oplus \mathcal{N} \vDash_{\langle g, h\rangle[x \mapsto\langle d, e\rangle]} \psi \\
& \Longleftrightarrow \mathcal{M} \oplus \mathcal{N} \vDash_{\langle g, h\rangle} \bar{\exists} x . \psi
\end{aligned}
$$

Case $\varphi \equiv \forall x . \psi$ the same passages as case $\varphi \equiv \bar{\exists} x . \psi$ apply here.

\section{Conclusions and Further Work}

In this paper we briefly presented the logic InqBQ and gave a proof of a conjecture formulated in [3], namely that the disjunction and existence properties hold for every classical theory. Moreover we found a generalization of the disjunction property and determined two classes of theories with interesting features connected to said property, namely theories with the $\oplus$ property and $q \mathbb{V}$-free theories.

To do this, a toolkit of model-theoretic constructions was developed in Section 3. These constructions proved to be effective instruments to study the semantics and entailment of InqBQ, and gave more insight into the mechanisms governing this logic. In view of this, we hope to have laid out in this paper the foundations of a model-theoretic approach to the study of InqBQ that could also lead to discover other meta-logical properties.

We end the paper with some remarks and some open questions.

\subsection{Remarks}

Throughout the whole paper, several definitions and results made a fundamental use of the varying identity of information models: to define $\mathbf{T} \mathcal{M}$; to define the product between a model and a set/a skeleton; to define $\mathcal{M}_{\Gamma}$; to combine models by means of the operator $\oplus$. One may wonder if we obtain a logic similar to InqBQ if we restrict our class of models to those that interpret identity rigidly, i.e. as the real identity in every world. It can be shown 
that in this alternative setup the disjunction and existence properties do not hold, and thus the result of this paper shows that indeed these two logics differ at least in their constructive content.

Another remark to make is that there is only one point in the paper where we made explicit use of the axiom of choice, namely in defining the model $\mathcal{M}_{\Gamma}$. All the other results and definitions do not require the use of this axiom.

\subsection{Open Questions}

To summarize the results of Section 5, we have the following classes of theories

TC: the class of truth-conditional theories.

$\mathrm{Cl}$ : the class of theories equivalent to a classical theory.

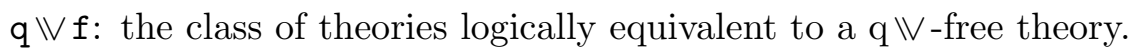

$\oplus \mathrm{P}: \quad$ the class of theories with the $\oplus$ property.

DP: the class of theories with the disjunction property.

and the following hierarchy

$$
\mathrm{TC} \underset{\text { Theorem 2.12 }}{=} \mathrm{Cl} \underset{\text { Remark 5.7 }}{\subsetneq} \mathrm{q} \backslash \mathrm{f} \underset{\text { Lemma 5.8 }}{\subseteq} \underset{\text { Lemma 5.4 }}{\subsetneq} \mathrm{DP}
$$

Some questions on this hierarchy remain open, the first being the following: do the classes $q \mathbb{V} \mathrm{f}$ and $\oplus \mathrm{P}$ coincide, as in the case of $\mathrm{TC}$ and $\mathrm{Cl}$ ?

A more conceptual question arises from the fact that we can divide the descriptions of the classes presented here into two kinds, semantic descriptions and syntactic descriptions. For example, the class TC is described semantically, as truth-conditionality of a theory $\Phi$ is a condition on the class of models of $\Phi$ (i.e., that it is closed under the operation $\biguplus$ for arbitrary families). On the other hand the class $\mathrm{Cl}$, although it coincides with TC, is described syntacticly as it is the class of theories of a certain fragment of the language (modulo equivalence).

In this regard DP is described neither purely syntactically, as we explicitly refer to the entailment relation, nor purely semantically, as the condition strongly focuses on inquisitive disjunctions. Are there syntactic and semantic conditions characterizing the class DP?

The same question can be asked for the classes $q \mathbb{V} f$ and $\oplus P$. Is there a semantic condition characterizing the class $q \mathbb{\vee} f$ ? Is there a syntactic condition characterizing the class $\oplus \mathrm{P}$ ? Of course a positive answer to the first question here would also resolve these issues. 
If we move to the existence property, the same question arise. Can we find a similar hierarchy for the existence property? And can we find syntactic and semantic characterizations for the existence property?

There are also more questions on InqBQ not directly or trivially related to the disjunction and existence properties that could be tackled using the tools and results presented in this paper. For example, is the logic compact? Are there any Löwenheim-Skolem type results? Is the logic axiomatizable, and in case of a positive answer, what is an axiomatization?

Acknowledgements. We gratefully acknowledge funding from the European Research Council (ERC, Grant Agreement Number 680220). We thank Ivano Ciardelli for extensive discussions, writing assistance and proofreading; Nick Bezhanishvili, Thom van Gessel and Floris Roelofsen for discussions and proofreading; Rosalie Iemhoff, Dick de Jongh, Juha Kontinen, Vít Punčochár̆, Jouko Väänänen and Jonni Virtema for discussions and comments. We would also like to thank the two anonymous reviewers for the useful comments.

Open Access. This article is distributed under the terms of the Creative Commons Attribution 4.0 International License (http://creativecommons.org/licenses/by/4.0/), which permits unrestricted use, distribution, and reproduction in any medium, provided you give appropriate credit to the original author(s) and the source, provide a link to the Creative Commons license, and indicate if changes were made.

\section{References}

[1] Abramsky, S., and J. VÄ̈̈nÄnen, From IF to BI: A tale of dependence and separation, Synthese 167(2): 207-230, 2009.

[2] Ciardelli, I., Inquisitive semantics and intermediate logics, Master's thesis, Institute for Logic, Language and Computation, University of Amsterdam, 2009.

[3] Ciardelli, I., Questions in logic, Ph.D. thesis, Institute for Logic, Language and Computation, University of Amsterdam, 2016.

[4] Ciardelli, I., Questions as information types, Synthese 195(1): 321-365, 2018.

[5] Ciardelli, I., J. GroenendiJk, and F. Roelofsen, Inquisitive semantics: A new notion of meaning, Language and Linguistics Compass 7(9): 459-476, 2013.

[6] Gabbay, D., V. Shehtman, and D. Skvortsov, Quantification in Non-classical Logics, Studies in Logic and Foundations of Mathematics, Elsevier, 2009.

[7] Hodges, W., Compositional semantics for a language of imperfect information, Logic Journal of IGPL 5(4): 539-563, 1997.

[8] Hodges, W., A Shorter Model Theory, Cambridge University Press, New York, NY, USA, 1997.

[9] Hodges, W., Some Strange Quantifiers, Springer Berlin Heidelberg, Berlin, Heidelberg, 1997, pp. 51-65. 
[10] Roelofsen, F., Algebraic foundations for the semantic treatment of inquisitive content, Synthese 190(1): 79-102, 2013.

[11] YAng, F., and J. VÄÄnÄnen, Propositional logics of dependence, Annals of Pure and Applied Logic 167(7): 557-589, 2016.

\author{
G. Grilletti \\ Institute for Logic, Language and Computation \\ Universiteit van Amsterdam \\ Science Park 107 \\ 1098 XG Amsterdam \\ The Netherlands \\ grilletti.gianluca@gmail.com
}

